ISSN: 0514-7336 — ISSN electrónico: 2386-3943

DOI: https://doi.org/10.14201/zephyrus2021873361

\title{
LA DECORACIÓN CERÁMICA EN EL NEOLÍTICO DE KAF TAHT EL-GHAR (TETUÁN, MARRUECOS). CAMPAÑA AGRIWESTMED 2012
}

\section{Pottery decoration in the Neolithic of Kaf Taht el-Ghar (Tetouan, Morocco). AGRIWESTMED Works 2012}

Rafael M. Martínez SÁnchez*, Juan Carlos Vera Rodríguez**, Guillem PÉrez Jordì ${ }^{* * *}$ y Leonor Peña-Chocarro****

* Dpto. de Historia. Facultad de Filosofía y Letras. Univ. de Córdoba. Plaza del Cardenal Salazar, 3. 14071, Córdoba.Correo-e: rmmartinez@uco.es. ID ORCID: https://orcid.org/0000-0002-8947-117X

** Dpto. de Historia, Geografía y Antropología. Facultad de Humanidades. Univ. de Huelva. Campus de El Carmen. Avda. Fuerzas Armadas, s/n. 21071 Huelva. Correo-e: vera@uhu.es. ID ORCID: http://orcid.org/00000002-5989-2694

*** Dpto. de Prehistoria, Arqueología e H. a Antigua. Univ. de Valencia. Avda. Blasco Ibáñez, 28. 46010 Valencia. Correo-e: guillem.perez@uv.es.ID ORCID: https:/lorcid.org/0000-0003-1459-0219

**** Instituto de Historia-CSIC. C/ Albasanz, 26-28. 28037 Madrid. Correo-e: leonor.chocarro@csic.es. ID ORCID: https://orcid.org/0000-0002-7807-8778

Recepción: 19/11/2020; Revisión: 4/02/2021; Aceptación: 1/05/2021

Resumen: Presentamos el estudio de los restos cerámicos de la fase neolítica de KafTaht el-Ghar (Mechrouha, Tetuán, Marruecos), enfocado principalmente en el análisis de técnicas y patrones decorativos. Ello cuenta con el interés ańadido de corresponder, en su fase antigua, a las cerámicas asociadas a las primeras evidencias de agricultura y ganadería en el Magreb Occidental, encuadradas en el tercer cuarto del vi milenio cal AC. En dichas decoraciones dominan impresiones variadas, en particular las realizadas con concha estriada y lisa, peine y de cypraea. El uso de tales técnicas podría incluir estas primeras producciones cerámicas dentro del mundo impresso-cardial de la Europa mediterránea centro-occidental. A una fase avanzada, atribuida al Neolítico Medio, corresponde un conjunto de cerámicas con fuertes analogías y conexiones con la denominada Achakar Ware, de rasgos técnico-estilísticos marcadamente diferenciados. Entre estos se cuenta el uso de engobe colorante y la aplicación de impresiones con roulette cordada, lo que parece relacionar estas ultimas producciones con tradiciones propias del actual territorio del Sahara.

Palabras clave: Península Tingitana; cerámica neolítica; patrones técnico-estilísticos; decoración impresa; engobe.

АвSTRACT: Here we present the study of the pottery remains from the Neolithic phase of Kaf Taht el-Ghar (Mechrouha, Tetouan, Morocco), focused on the analysis of decorative patterns and techniques. In its early phase, its association with the first evidence of agriculture and livestock in the western Maghreb, framed in the third quarter of the $6^{\text {th }}$ millennium $\mathrm{BC}$, is noteworthy. A variety of impressions are dominant in the decorations, those made with striated and smooth shells, combs, and cowry. The use of these techniques could include 
34 R. M. Martínez Sánchez, J. C. Vera Rodríguez, G. Pérez Jordà y Leonor Peña-Chocarro / La decoración cerámica en el Neolítico...

these first ceramic productions within the impressa-cardial complex of central-western Mediterranean Europe. An advanced phase, assigned to the Middle Neolithic, includes a group of ceramics with strong analogies and connections with the so-called Ashakar Ware, of notably different technical and stylistic features. These include the use of coloured slip and the application of roulette-corded impressions, suggesting a link with former traditions specific to the Sahara territory.

Key words: Tingitanian Peninsula; Neolithic pottery; technical-stylistic patterns; impressed decoration; slip.

\section{Introducción}

\subsection{Las primeras cerámicas del Magreb occidental}

El origen y aparición de la cerámica en el Magreb ha alimentado un encendido debate, donde convergen problemáticas particulares aún no resueltas. Entre todas ellas, el principal punto clave versa en considerar si, como en el caso de la Europa Mediterránea, la cerámica constituye una innovación más incluida dentro del denominado 'Paquete Neolítico' desarrollado en el suroeste de Asia y difundida en progresión a partir del vir milenio desde el $\mathrm{N}$ de Siria y Anatolia al resto del Mediterráneo. La postura que podríamos considerar contraria reafirma esta tecnología como una innovación presente desde los inicios del Holoceno en la región, como ha sido probado en diversos puntos del actual territorio del Sahara y que condujo a acuñar el término 'Neolítico Saharo-Sudanés' (Camps, 1969, 1974; Camps-Fabrer, 1967), en cuyo caso se ha defendido la existencia de una neolitización de raigambre autóctona para el Magreb noroccidental. Parte de dicho enfoque ha servido para reforzar las posiciones de diversos autores para esta región (Daugas et al., 2008; El Idrissi, 2012a), definiendo dos tradiciones

\footnotetext{
GPJ es miembro de GRAM-GIUv2015-222 y LPCH lo es de GI "Paleoeconomía y Subsistencia de las Sociedades Preindustriales". La excavación y el procesamiento de los materiales arqueológicos de Kaf Taht el-Ghar ha sido financiado por el European Research Council a través del Proyecto AGRIWESTMED (Origins and spread of agriculture in the western Mediterranean region) coordinado por L. Peña-Chocarro, con la colaboración del INSAP (Institut National des Sciences de l'Archéologie et du Patrimoine). Agradecemos a los colegas de dicha institución Y. Bokbot, A. El Idrissi y F. Amani su apoyo durante la ejecución de la campańa arqueológica. Este trabajo está dedicado a la memoria de Mustapha Nami y Lydia Zapata.
}

representadas por un Neolítico de tradición capsiense -en particular en el Tell argelino- (Roubet, 1979) y otra mediterránea, caracterizada por cerámicas impresas y cardiales (Camps, 1974), lo que también influiría en la contextualización del fenómeno en el Magreb Oriental y Túnez (Mulazzani et al., 2016).

Así, y aunque en los últimos diez años se han incorporado nuevas perspectivas y enfoques a la visión general del Neolítico en el Magreb occidental (Linstädter et al., 2012; Martínez et al., 2018a y b), el enfoque que podríamos llamar tradicional propone la conjunción de una tradición de tipo telliano-argelino, otra de tipo sahariano, y otra de origen mediterráneo, ligada a la tradición impressa-cardial (El Idrissi, 2012a). En este caso el término cardial se emplea en función de la decoración cerámica realizada con concha estriada, independientemente del aspecto cronocultural con el que recientemente se ha resignificado el término en el Mediterráneo occidental europeo (Manen et al., 2019) y de su uso para definir en conjunto las primeras cerámicas del norte de Marruecos ${ }^{2}$. La presencia de estos conjuntos cerámicos se constata en la región septentrional marroquí, entre el Temara (El Idrissi, 2012bc), el piedemonte del Atlas Medio (Martínez et al., 2018b), y el área de Melilla-Alhucemas (Nekkal et al., 2015; Linstädter y Wagner, 2013). En este sentido podemos apreciar cierta homogeneidad entre los yacimientos de Tánger y Cabo Achakar, Oued Tahadart, Gar Cahal, Kef Boussaria y Kaf Taht elGhar (Jodin, 1958-1959; Gilman, 1975; El Idrissi, 2008; Tarradell, 1954, 1955, 1957-1958; Vijande et al., 2011), visible no solo entre las cerámicas impresas con concha estriada, sino también entre

2 El Idrissi, A.: Néolithique ancien du Maroc Septentrional dans son contexte régional. Tesis doctoral inédita presentada en 2001 en el Institut National de Sciences de l'Archéologie et du Patrimoine de Rabat. 
otros grupos cerámicos propios de esta fase antigua, a la que hemos denominado Clúster Tingitano (Fig. 1).

\subsection{La cueva de Kaf Taht el-Ghar (KTG)}

Esta cavidad constituye una amplia y compleja caverna dispuesta en torno a una sala principal de unos $12 \mathrm{~m}$ de anchura por 30 de profundidad, a la que se accede por una entrada de fácil acceso orientada al No. Se encuentra en la vertiente oriental de la Península Tingitana, a unos $8 \mathrm{~km}$ al SE de la ciudad de Tetuán y a $410 \mathrm{msnm}$, siguiendo las coordenadas 35030'34,75” N y 5o 19'48,68” O. Se sitúa aproximadamente a 9,2 km de la actual línea de costa, junto al cabo Ras Mazarí, si bien enclavada en un medio montañoso sobre un farallón rocoso que constituye el extremo NE del Djabal Gharghez.

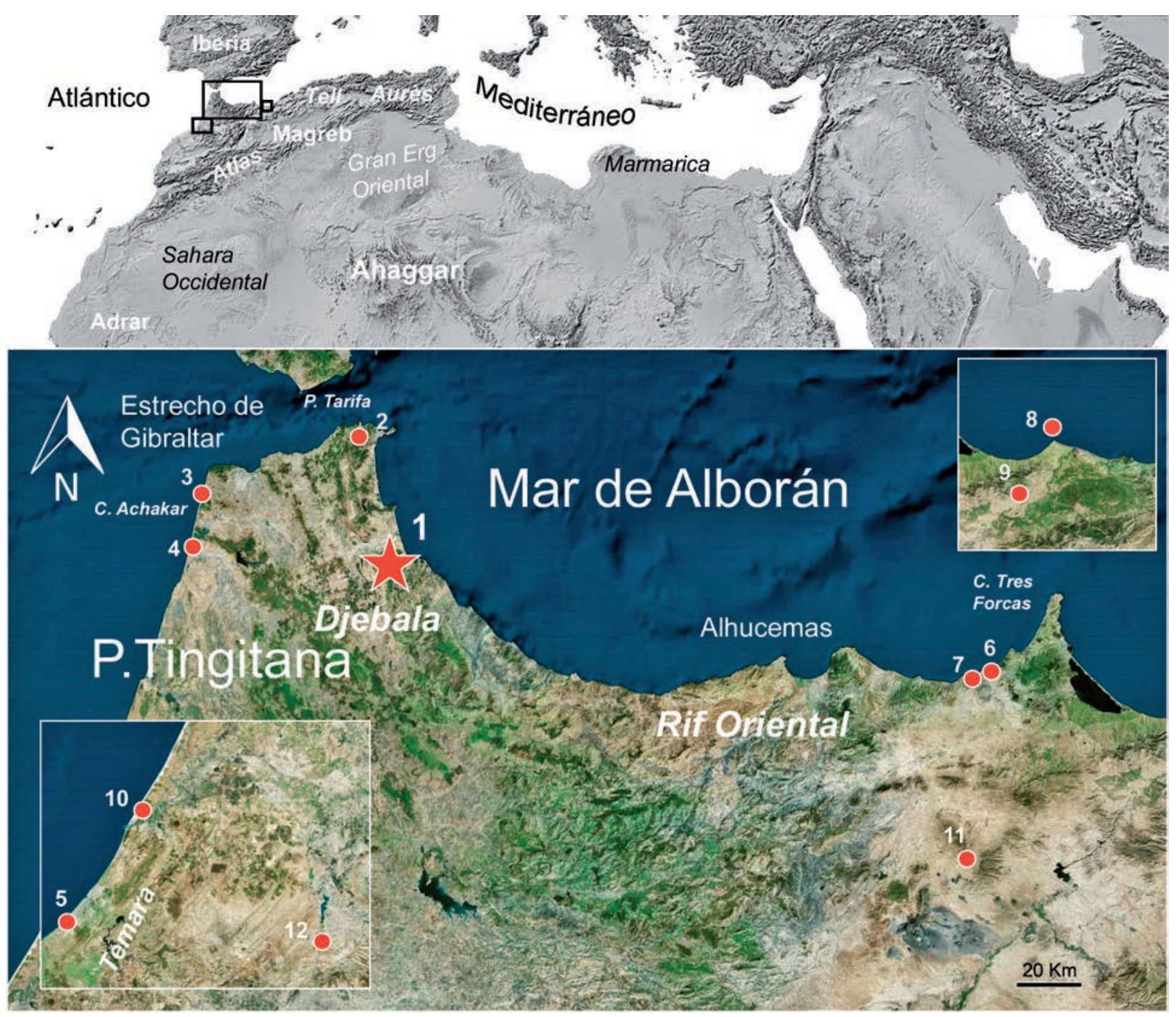

FIG. 1. Arriba: la Peninsula Tingitana y Riforiental en el contexto del norte de África y del mar Mediterráneo. Abajo: yacimientos mencionados en el texto: 1) KTG y Kef Boussaria; 2) Gar Cahal; 3) yacimientos de Cabo Achakar: Magharat el-Khil, Les Idoles, Mugaret el Aliya y Mugaret es Saifiya; 4) Oued Tahadart; 5) El Harhoura; 6) Ifri Ouzabour; 7) Ifri Oudadane; 8) Zafrin; 9) Ifri Etsedda; 10) El Mnasra; 11) Hassi Ouenzga; 12) Ifri n’Amr Ou Moussa. 
La cueva fue excavada por vez primera por M. Tarradell en otońo de 1955, quien publicó dos artículos muy preliminares (Tarradell, 1955, 19571958). Dado el carácter de las excavaciones y la metodología de la época, la secuencia obtenida fue bastante esquemática, sintetizada en una estratigrafía de cuatro niveles. El Nivel I -Estratos 1 y 2- correspondería a ocupaciones históricas -medievales y modernas-. El Nivel ir correspondería al Estrato 3, con elementos protohistóricos -identificados como púnicos-y de la Prehistoria Reciente -Campaniforme y Edad del Bronce-. El Nivel III, con los Estratos 4 y 5 , correspondería a una rica fase neolítica, con cerámicas decoradas impresas, destacando aquellas con impresiones cardiales de gran diversidad en formas y tipos. Por último, el Nivel Iv, con una matriz arenosa de coloración rojiza que Tarradell (1955) consideró casi estéril, con 'sílex poco típicos' y restos óseos muy carbonatados, definía la fase más antigua, directamente apoyada sobre la roca basal.

Décadas más tarde, la cueva volvió a ser investigada en el marco de la cooperación entre la Mission Préhistorique et Paléontologique Française au Maroc -MPPFM- y el INSAP - Programme Néolithique du Littoral Nordatlantique-. Estas se iniciaron en 1984 y 1987 bajo la dirección de J. P. Daugas, J. P. Raynal y A. Ballouche. Durante estos trabajos, en los que se despejaron hasta 42 UUEE, se pudo constatar por primera vez la existencia de una rica fase epipaleolítica previa, confirmando a su vez la presencia de ocupaciones postneolíticas -cerámica campaniforme- e históricas. La ocupación neolítica quedó dividida entre una fase inicial, que se relacionó con fechas sorprendentemente antiguas, y una posterior fase cardial. Esta última quedaría dividida en dos períodos diferenciados a partir de estilos y decoraciones cerámicas, por un lado, y dataciones absolutas efectuadas por radiocarbono y termoluminiscencia, por otro. Los resultados relativos a la fasificación propuesta y su cronología fueron apareciendo en trabajos generales de revisión (Daugas et al., 2008; El Idrissi, 2012a), mientras que la cerámica correspondiente al Neolítico Antiguo fue abordada de manera conjunta junto a otras colecciones de la Península Tingitana en una tesis doctoral ${ }^{3}$.

\subsection{El proyecto AGRIWESTMED en KTG}

La última de las intervenciones realizadas, cuyos materiales cerámicos presentamos en este trabajo, se efectuó en el marco de un convenio de colaboración científica firmado entre el CSIC español y el INSAP marroquí, bajo el paraguas del proyecto ERC AGRIWESTMED, dirigido por uno de nosotros - $\mathrm{LPCH}-$. Esta consistió en la realización de un total de cuatro sondeos estratigráficos de escasa extensión, dos de ellos contiguos, practicados en la zona central de la gran sala principal de la cavidad. Aunque la zona había sido ya explorada en las campañas anteriores y se encontraba muy afectada por derrumbe de perfiles y alteraciones postdeposicionales recientes, nos propusimos localizar el testigo de dirección E-O que separaba los Sectores 1-4 y 2-3 de las intervenciones de 1989-1994 (Fig. 2). El objetivo fundamental, más que proceder a la recuperación de un abundante registro material, fue el establecimiento de un marco cronológico amplio y de alta resolución a partir de dataciones absolutas sobre elementos orgánicos de vida corta, resolviendo a la vez algunos de los problemas de falsificación planteados en las campañas anteriores. Aprovechando la anterior sectorización mediante cuadrículas alfanuméricas, el código 26 fue respetado como identificador primario de la ubicación de los diferentes sondeos, siendo denominados de E a O: 26 A-B, 26 G, 26 H-I y 26 J-K.

La nueva lectura estratigráfica (Martínez et al., 2021) confirmó una ocupación epipaleolítica intensa, situada entre el Drías Reciente y el evento 8.2 cal AC. Dicha fase se encuentra representada por las UUEE 1105 de 26 A-B y Ampliación, 1208 a 1209-3 de 26 H-I y 26 J-K y las UUee 1003-2 a 1006 Base del Sondeo 26 G. Sin evidencias de domesticación, el conjunto material se caracteriza por una relativamente rica industria lítica laminar, con predominio de dorsos y marcada ausencia de cerámica.

3 El Idrissi, op. cit. n. 2. 

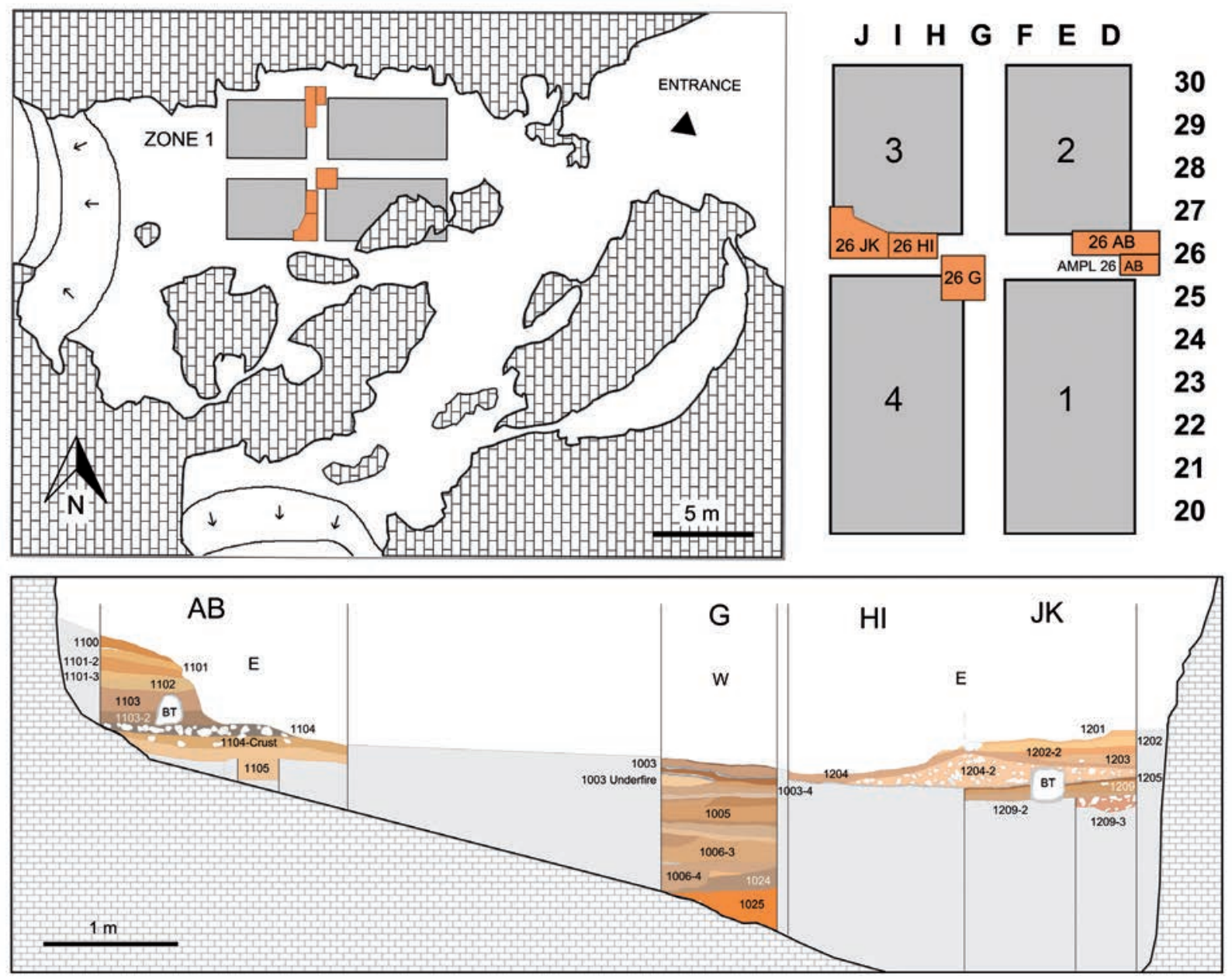

FIG. 2. Arriba: ubicación de los sondeos realizados en KafTaht el-Ghar dentro del proyecto AGRIWESTMED en la Zona 1 del vestíbulo, sobre la cruceta-testigo (26) conservada de las excavaciones de las dos últimas décadas del s. XX. Abajo: estratigrafía obtenida, representando los perfiles $E$ en $A B, H I$ y $J K, y$ W en $G$ (representado especularmente).

Tras un evento de abandono o ausencia de ocupación en la secuencia despejada -Unidades 1003 Costra $=1205$ y $1104-$, la ocupación se reanuda con un primer horizonte neolítico, correspondiente a la sucesión estratigráfica presente entre las UUEE 1103-1 y 1104 Concreción de 26 A-B y Ampliación $\mathrm{y}$ entre las UUEe 1003-1 = 1204-1 y 1003 Costra = 1205 de los sondeos 26 G, 26 H-I y 26 J-K. Será esta fase la que venga a inaugurar la presencia de materiales cerámicos, más allá de algunos elementos intrusivos hallados en el nivel intermedio, correspondiente al hiato, y en el interior de bioturbaciones y madrigueras, constantes a lo largo de la secuencia, y que también afectan a la fase epipaleolítica. Esta primera fase neolítica se encuadra a lo largo de la segunda mitad del vi milenio -c. 5450-5000 cal AC-, estando caracterizada por los primeros cultivos -cereales y leguminosas como Triticum dicoccum, Triticum aestivum/durum, Vicia faba, Pisum sativum (Morales et al., 2016)-, así como por la presencia de caprinos domésticos-Ovis aries-.

La fase correspondiente al Neolítico Medio abarca las UUEe 1101-3 y 1102 de los sondeos 26 A-B y Ampliación y las 1202-1 a 1203 de 26 H-I y $26 \mathrm{~J}-\mathrm{K}$, algo menos ricas en restos artefactuales. La única datación disponible -Beta-331986, UE 
1202-2-, sobre Triticum dicoccum, situaría dicha ocupación en la segunda mitad del v milenio cal AC -c. 4200 cal AC-, si bien no podemos descartar la existencia de elementos propios de momentos algo más antiguos quizá resultado de un palimpsesto de ocupaciones producidas a lo largo de dicho milenio, viendo la proporción de elementos residuales -elementos cerámicos indudables de la primera fase neolítica- en estos estratos (Fig. 3).

Las fases ulteriores de la Prehistoria Reciente -Campaniforme y Edad del Bronce- solo han podido insinuarse a partir de algunos fragmentos cerámicos de ese aspecto en niveles superiores, donde la presencia de cerámicas a torno y otros elementos de escasa antigüedad han servido para considerar como histórica sensu lato la fase más reciente. En cualquier caso, la presencia de materiales propios del III milenio en forma de cerámica campaniforme ya fue atestiguada por la investigación precedente (Tarradell, 1955; Ramos et al., 2008).

\section{La cerámica neolítica de Kaf Taht el-Ghar}

\subsection{Material y métodos}

Este trabajo se centra en las cerámicas recuperadas en KTG dentro del proyecto AGRIWESTMED, un conjunto mucho más reducido que el recuperado a lo largo de los primeros trabajos (Tarradell, 1955, 1957-1958; Ramos et al., 2008) y en las campañas de 1989-1994 dirigidas por la MPPFM 4 , a los que no hemos tenido acceso. Por ello, debe ser considerado como un trabajo parcial enfocado a la comprensión de la evolución técnica y estilística de las producciones cerámicas en el yacimiento, a su vez ligado a los trabajos de muestreo y obtención de la secuencia.

Hemos partido en primer lugar de la fotografía de la práctica totalidad de las piezas decoradas y de una gran parte de aquellos fragmentos con forma total o parcialmente reconstruible. Igualmente, dichos fragmentos y los que presentaban algún tipo de decoración fueron dibujados usando rapidógrafo de tinta y agrupados en composiciones mediante

4 El Idrissi, op. cit. n. 2. programas de tratamiento de imagen -Photoshop cs3-. A diferencia de las secciones de los fragmentos, que fueron mantenidas, el dibujo sombreado de las superficies fue finalmente sustituido por las propias fotografías de detalle en cuanto a las piezas decoradas, a efectos de una mayor resolución y objetividad en el aparato gráfico en la publicación. Junto a ello, se procedió a la contabilización y desglose íntegro del material en una base de datos, contando un registro por cada fragmento, el cual ha sido subdividido a su vez en campos diferenciados por tipo y técnica decorativa, región anatómica o forma en su caso, entre otras variables.

El estudio porcentual se ha centrado en el análisis de fragmentos (Manen, 2000). Aunque partimos de la base de considerar más representativo el análisis de los restos cerámicos en cuanto a su asociación a unidades vasculares o vasos individuales (Orton y Hughes, 2013; Oms, 2014), decidimos evitarlo debido a las limitaciones del registro obtenido. En este sentido, el escaso número de fragmentos recuperados frente a la colección existente procedente de intervenciones anteriores (Tarradell, 1954, 1955; y sobre todo en las de las décadas de 1980 y 1990$)^{5}$, hizo este enfoque poco recomendable, considerando el fuerte sesgo que ello implica. Así, resulta evidente la repetición de motivos y patrones idénticos para vasos distintos, como cabe observar en los ejemplares reconstruidos procedentes de las citadas campañas.

Entre las variables observadas incluimos la asignación 'anatómica' de cada fragmento. De este modo se han clasificado como base, galbo, cuello, borde o elementos de prensión y suspensión, asas y similares -en adelante EPS-. Debido a la existencia de niveles de cronología histórica en los estratos superficiales, inicialmente diferenciamos entre cerámica confeccionada a torno o a mano. Dada la escasa significación de los materiales procedentes de dichos niveles y su heterogeneidad -donde priman cerámicas impresas neolíticas residuales junto con material a torno y cerámicas pre- y protohistóricas de difícil adscripción-, finalmente optamos por prescindir de dichos conjuntos en este estudio.

5 El Idrissi, op. cit. n. 2. 


\begin{tabular}{|c|c|c|c|c|c|c|}
\hline FASES & & 26AB/26AB AMP & & $26 \mathrm{G}$ & & $26 \mathrm{HI} / 26 \mathrm{JK}$ \\
\hline \multirow{4}{*}{1} & \multirow{4}{*}{ HIS } & 1100 & & & & \\
\hline & & 1100 hogar & & & & \\
\hline & & $1101-1$ & & & & 1201 \\
\hline & & $1101-2$ & & & & \\
\hline \multirow{4}{*}{2} & \multirow{4}{*}{ NM } & & & & & $1202-1$ \\
\hline & & & & & & $1202-2$ \\
\hline & & $1101-3$ & & & & \\
\hline & & 1102 & & & & 1203 \\
\hline \multirow[b]{2}{*}{3} & \multirow[b]{2}{*}{$\mathrm{NA}$} & $1103-1$ & & \multirow[t]{2}{*}{$1003-1$} & & $1204-1$ \\
\hline & & $1103-2$ & & & & $1204-2$ \\
\hline 4 & TRANS & 1104 & & 1003 costra & & 1205 \\
\hline \multirow{20}{*}{5} & \multirow{20}{*}{ EPI } & 1104 concr & & $1003-2$ & & \multirow[t]{3}{*}{1208} \\
\hline & & \multirow[b]{3}{*}{1105} & & 1003 hogar & & \\
\hline & & & & 1003 bajo hogar & & \\
\hline & & & & $1003-3$ & & $1209-1$ \\
\hline & & & & $1003-4$ & & $1209-2$ \\
\hline & & & & $1003-5$ & & \\
\hline & & & & 1003 suelo & & \\
\hline & & & & $1003-6$ & & $1209-3$ \\
\hline & & & & $1003-7$ & & \\
\hline & & & & 1004 & & \\
\hline & & & & 1005 & & \\
\hline & & & & 1019 & & \\
\hline & & & & $1006-1$ & & \\
\hline & & & $1006-2$ & & 1006 hogar & \\
\hline & & & & 1006 fosa & & \\
\hline & & & & $1006-3$ & & \\
\hline & & & & $1006-3$ cenizas & & \\
\hline & & & & $1006-4$ & & \\
\hline & & & & 1007 interfacies & & \\
\hline & & & & 1006 base & & \\
\hline \multirow{3}{*}{6} & \multirow{3}{*}{ PAL } & & & 1024 & & \\
\hline & & & & 1025 & & \\
\hline & & & & ROCA MADRE & & \\
\hline
\end{tabular}

FIG. 3. Secuencia estratigráfica por UUEE y sondeo, despejada en la intervención de 2012-AGRIWESTMED. 
40 R. M. Martínez Sánchez, J. C. Vera Rodríguez, G. Pérez Jordà y Leonor Peña-Chocarro / La decoración cerámica en el Neolitico...

\begin{tabular}{|c|c|c|c|}
\hline Grupo Principal & Cod. & TÉCNICA & INSTRUMENTO \\
\hline \multirow{8}{*}{ cardial } & $\mathrm{I} 1 \mathrm{~A}$ & impresiones simples verticales & \multirow{6}{*}{ cardium o valva estriada } \\
\hline & І1в & basculante vertical & \\
\hline & I1C & impresiones simples con la concha oblicua u horizontal & \\
\hline & Ild & $\begin{array}{l}\text { impresiones superpuestas con la concha oblicua u } \\
\text { horizontal }\end{array}$ & \\
\hline & I1E & basculaciones con la concha oblicua u horizontal & \\
\hline & $\mathrm{I} 1 \mathrm{~F}$ & arrastre cardial & \\
\hline & $\mathrm{I} 2 \mathrm{~A}$ & impreso vertical simple & \multirow{2}{*}{ valva lisa } \\
\hline & $\mathrm{I} 2 \mathrm{~B}$ & basculante vertical (rocker) & \\
\hline \multirow{3}{*}{$\begin{array}{l}\text { acanaladas } \\
\text { (Channeled Ware) }\end{array}$} & $\mathrm{I} 3 \mathrm{~A}$ & impresiones verticales en forma de coma & \multirow{3}{*}{ canal sifonal de cypraea } \\
\hline & II $1 \mathrm{~A}$ & acanaladuras cortas & \\
\hline & II1в & acanaladuras largas & \\
\hline \multirow{4}{*}{ a peine } & $\mathrm{I} 4 \mathrm{~A}$ & impresiones simples verticales & \multirow{4}{*}{ peine/instrumento de puntas múltiples } \\
\hline & $\mathrm{I} 4 \mathrm{~B}$ & impreso oblicuo concatenado & \\
\hline & $\mathrm{I} 4 \mathrm{C}$ & impresiones simples oblicuas & \\
\hline & $\mathrm{I} 4 \mathrm{D}$ & peine basculante & \\
\hline \multirow{9}{*}{ impresas } & $15 \mathrm{~A}$ & impresiones verticales & \multirow{6}{*}{ otros instrumentos } \\
\hline & $\mathrm{I} 5 \mathrm{~B}$ & impreso oblicuo lineal & \\
\hline & I7A & impresiones curvas & \\
\hline & $\mathrm{I} 9 \mathrm{~A}$ & impresiones con instrumento indeterm. & \\
\hline & I9в & impresión pivotante & \\
\hline & $\mathrm{I} 9 \mathrm{C}$ & impresión oblicua superpuesta & \\
\hline & $\mathrm{I} 6 \mathrm{~A}$ & ungulación & \multirow{3}{*}{ uñas/dedos } \\
\hline & I6B & pinzado & \\
\hline & I6C & impresiones digitales & \\
\hline \multirow{2}{*}{ incisas } & II $2 \mathrm{~A}$ & incisión corta & \multirow{2}{*}{ punzón/instrumento apuntado } \\
\hline & $\mathrm{II} 2 \mathrm{~B}$ & incisión larga & \\
\hline \multirow{2}{*}{ modeladas } & III2 & cordón & \multirow{2}{*}{ aplicaciones plásticas } \\
\hline & III3 & mamelón/diversos & \\
\hline \multirow{3}{*}{ tipo Achakar } & $\mathrm{I} 9 \mathrm{E}$ & impresiones en cuentas de rosario & roulette de cuerda enrollada \\
\hline & IV1 & engobe rojo o beige & \multirow{2}{*}{ polvo mineral/engobe } \\
\hline & IV3 & incrustación & \\
\hline
\end{tabular}

FIG. 4. Tabla de las decoraciones por códigos; desglose de las técnicas decorativas e instrumento empleado.

Los aspectos técnico-analíticos referidos a la composición de pastas y desgrasantes (Stempfle et al., 2018) no han sido tenidos en consideración para este trabajo, en primer lugar, dada la necesidad de analíticas físico-químicas y petrológicas que permitan contrastar las observaciones macroscópicas de forma objetiva y, en segundo lugar, al no disponer del tiempo e instrumental necesario en el intervalo consagrado al estudio del material. Otros aspectos, como los acabados y el tratamiento de las superficies, no han sido incluidos al depender en gran medida del estado de conservación de las piezas y la extensión de la decoración que presentan. La adición de pintura o fundamentalmente engobe de color, normalmente rojo o beige, ha sido incluida dentro de las decoraciones, al agrupar una técnica tan solo observada en cerámicas correspondientes al Neolítico Medio, características de la conocida como cerámica de tipo Achakar-o Ashakar Ware-.

El estado de conservación muy fragmentario de la mayor parte de los elementos ha afectado directamente a la aproximación morfológica de los recipientes. La clasificación por formas cerámicas no ha sido fácil, reduciéndose en formas ovoides de borde no diferenciado y formas de leve cuello estrangulado y labio exvasado, ambas con diferentes variantes y tamaños. En este sentido, el repertorio de formas existentes puede ser valorado con una mayor resolución gracias a la observación de fragmentos de mayor tamaño o parcialmente reconstruidos 
procedentes de excavaciones anteriores, donde el segundo tipo se asocia con frecuencia a formas de tipo anfórico, con base apuntada. Por su parte, en el conjunto propio del Neolítico Medio, y en concreto los fragmentos atribuidos a las Ashakar Ware, apenas se han podido identificar algunas formas de paredes rectas y base plana.

Para la definición de los tipos decorados, desarrollamos una tabla de clasificación estructurada estrictamente en torno a las técnicas usadas en la decoración cerámica, evitando la descripción y clasificación de los diseńos, estructura compositiva y sintaxis decorativa. Dicha tabla, complementaria a la utilizada en un trabajo anterior (Martínez et al., 2018b), se ha completado con diversos códigos alfanuméricos que han tenido en cuenta el tipo de instrumento y su posición y, en algunos casos, la extensión de la técnica empleada (Figura 4). Como paso previo nos centramos en reproducir experimentalmente cada una de estas técnicas, a fin de evaluar la fiabilidad de las hipótesis avanzadas respecto a la forma de decorar la cerámica. Los resultados despejados por dicha aproximación experimental han servido para la descripción crítica de las técnicas decorativas implicadas -impresiones de valvas de moluscos, conchas de ciprea, peine, impresiones con los dedos, etc.-- Cada uno de estos códigos se ha agrupado en conjuntos más amplios, referidos al grupo o tipo cerámico definido en la investigación precedente, asociados a usos culturales o ubicación cronológica concreta (Fig. 5). Como adelantamos, la dificultad de definir motivos y composiciones

\section{Grupo Cardial}

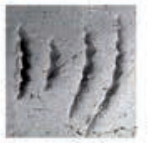

I1A

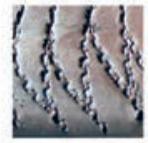

I1B

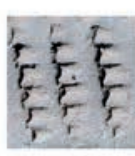

I1C

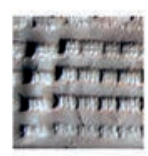

I1D

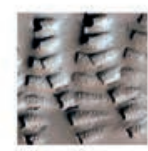

I1E

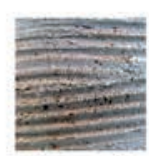

I1F

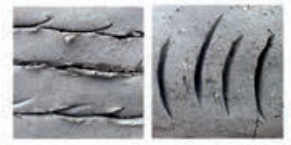

I2A

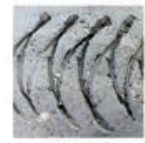

I2B
Grupo Acanalada

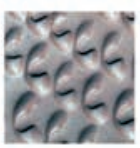

I3A

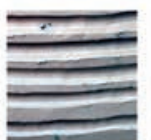

II1A

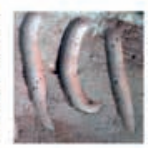

II1B

\section{Grupo Peine}

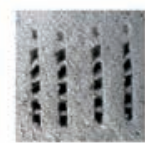

$14 \mathrm{~A}$

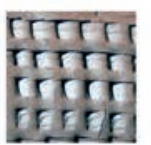

I4B

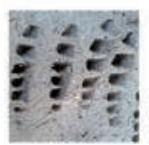

I4C

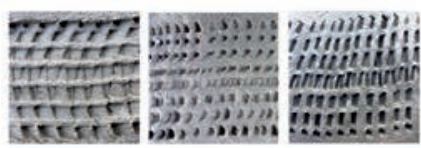

I4D

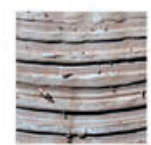

II3B

\section{Grupo Impresa}

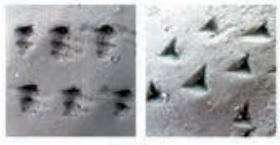

I5A

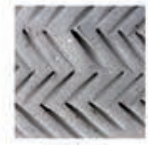

I5B

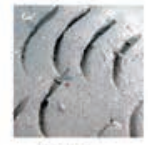

I7A

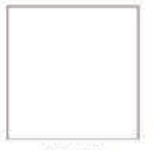

I9A

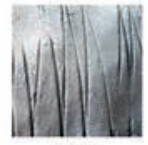

I9B

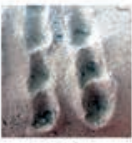

I9C

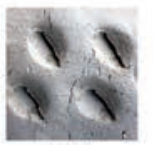

I6A

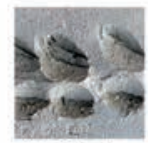

I6B

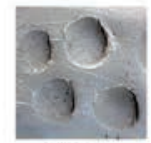

I6C
Grupo Modelada

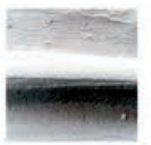

III2

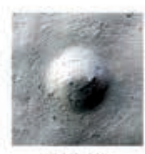

III3
Grupo Incisa

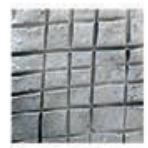

II2A

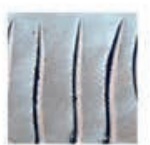

II2B

\section{Grupo Achakar}

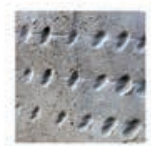

I9E

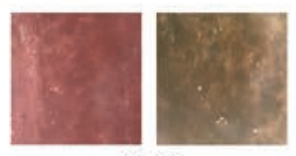

IV1

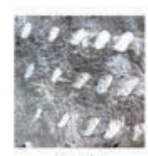

IV3

FIG. 5. Técnicas decorativas identificadas por grupos en la cerámica neolitica de KTG y reproducidas a partir de una batería experimental; cf. descripción de códigos en la Fig. 4. 
sobre las que se articularían las decoraciones (Bernabeu et al., 2011) ha resultado otra de las limitaciones derivadas de la amplia fragmentación, por lo que, frente a la específica de las técnicas decorativas y grupos temáticos, dicha variable solo se ha considerado para una valoración general del conjunto.

Para la definición de estos grupos cerámicos, hemos partido de la clasificación de A. Gilman desarrollada para los yacimientos del área de Tánger y Cabo Achakar, considerando ser la más racional al compartir aspectos formales y cronológicos vinculados directamente a su técnica y decoración sensu stricto. Dichos grupos principales se dividen entre cardial -decorada fundamentalmente con concha estriada-, acanalada -Channeled Ware-, a peine, impresa -no cardial-, incisa y modelada, incluyendo el grupo visiblemente posterior, que combina engobe rojo e impresión a roulette, como es la Ashakar Ware (Gilman, 1975).

\begin{tabular}{|c|c|c|c|c|c|}
\hline Cronología & UUEE & $\begin{array}{c}\text { N. }{ }^{\circ} \\
\text { DECOR. }\end{array}$ & $\begin{array}{c}\% \\
\text { DECOR. }\end{array}$ & $\begin{array}{c}\text { N. }^{\circ} \\
\text { NO DECOR. }\end{array}$ & $\begin{array}{c}\% \\
\text { NO DECOR. }\end{array}$ \\
\hline \multirow{5}{*}{$\begin{array}{l}\text { Neolítico } \\
\text { Medio }\end{array}$} & $1101-3$ & 5 & 22 & 18 & 78 \\
\hline & 1202 & 17 & 40 & 26 & 60 \\
\hline & $1202-2$ & 9 & 41 & 13 & 59 \\
\hline & 1102 & 30 & 25 & 91 & 75 \\
\hline & 1203 & 3 & 20 & 12 & 80 \\
\hline \multirow{6}{*}{$\begin{array}{l}\text { Neolítico } \\
\text { Antiguo }\end{array}$} & 1103 & 170 & 61 & 110 & 39 \\
\hline & 1204 & 26 & 43 & 35 & 57 \\
\hline & $1103-2$ & 24 & 53 & 21 & 47 \\
\hline & 12042 & 34 & 34 & 66 & 66 \\
\hline & $1003-1$ & 2 & 17 & 10 & 83 \\
\hline & 1104 & 2 & 12 & 14 & 88 \\
\hline Totales & & 322 & 44 & 416 & 56 \\
\hline
\end{tabular}

FIG. 6. Número y porcentaje entre fragmentos decorados y no decorados obtenidos de las fases neoliticas de $K T G-2012$.

\begin{tabular}{|l|c|c|c|c|}
\hline \multicolumn{1}{|c|}{ Grupos } & $\begin{array}{c}\text { N. }^{\circ} \\
\text { NeOLÍtico } \\
\text { AnTiguo }\end{array}$ & $\begin{array}{c}\% \\
\text { NeOLÍtico } \\
\text { Antiguo }\end{array}$ & $\begin{array}{c}\text { N. }{ }^{\circ} \\
\text { NeOLÍtico } \\
\text { Medio }\end{array}$ & $\begin{array}{c}\% \\
\text { Neolítico } \\
\text { Medio }\end{array}$ \\
\hline cardial & 94 & 33,10 & 22 & 31,88 \\
\hline acanalada & 72 & 25,35 & 16 & 23,19 \\
\hline peinada & 24 & 8,45 & 2 & 2,90 \\
\hline impresa & 50 & 17,61 & 7 & 10,14 \\
\hline modelada & 18 & 6,34 & 4 & 5,80 \\
\hline incisa & 26 & 9,15 & 6 & 8,70 \\
\hline Achakar & 0 & 0 & 12 & 17,39 \\
\hline
\end{tabular}

FIG. 7. Número y porcentaje por grupos decorados entre los fragmentos de las fases neoliticas de KTG-2012.

\subsection{Resultados}

El número total de fragmentos cerámicos asciende a 844, si bien 66 corresponden a niveles históricos-UUEE 1100, 1101-1, 1101-2, 1201-, de los que 12 constituyen ejemplos a torno. Por su parte, 40 proceden de superficie, limpiezas sin atribución estratigráfica y del interior de madrigueras o bioturbaciones que afectan a la secuencia en distintos tramos. Por ello, nos centraremos solo en el conjunto correspondiente a los niveles del Neolítico Antiguo -uUeE1103-1, 1103-2, 1003-1, 1204-1, 1204-2-; horizonte transicional del Epipaleolítico/

Neolítico, asociado a la ue 1104 y de donde procede un reducido grupo de fragmentos que hemos asimilado al Neolítico Antiguo, y Neolítico Medio -UUEe 1101-3, 1102, 1202-1, 1202-2 y 1203-. Así, el total del conjunto abordado alcanza los 738 fragmentos, valiéndonos de las piezas no atribuidas o sin adscripción estratigráfica solo a efectos comparativos.

\subsubsection{Neolítico Antiguo}

Este conjunto se compone de 514 fragmentos, divididos de forma casi equilibrada -a un $50 \%-$ 
entre decorados y no decorados -258 y 256 respectivamente-. Dentro de las piezas decoradas, las incluidas dentro del grupo Cardial son las más numerosas, con 94 fragmentos que cuentan con una o varias técnicas, individualmente o en asociación.
Frente a otras definiciones del grupo Cardial, que incluyen diversas técnicas -el grupo de las acanaladas, otras impresas o incluso algunas desprovistas de decoración- dentro un enfoque principalmente cronológico (El Idrissi, 2012c), en nuestra

\begin{tabular}{|c|c|c|c|c|c|c|c|c|c|c|c|c|}
\hline \multirow{2}{*}{\multicolumn{2}{|c|}{ DECORACIÓN/FASE }} & MN & & & & & $\mathrm{EN}$ & & & & & \\
\hline & & $1101-3$ & 1202 & $1202-2$ & 1102 & 1203 & 1103 & 1204 & $1103-2$ & $1204-2$ & 1003 & 1104 \\
\hline \multirow[t]{9}{*}{ cardial } & $\mathrm{I} 1 \mathrm{~A}$ & - & 1 & 2 & 2 & - & 3 & 2 & - & 5 & - & - \\
\hline & І1в & - & 2 & - & 3 & - & 7 & 2 & - & - & - & - \\
\hline & IlC & - & - & 2 & - & - & 7 & 1 & 1 & 5 & 2 & - \\
\hline & $\mathrm{I} 1 \mathrm{~F}$ & - & 3 & 1 & 3 & 1 & 41 & 4 & 2 & 1 & - & - \\
\hline & $\mathrm{I} 1 \mathrm{E}$ & - & - & - & - & - & 2 & 2 & 1 & - & - & - \\
\hline & I1D & - & - & - & - & - & 4 & - & - & 1 & - & - \\
\hline & $\mathrm{I} 2 \mathrm{~A}$ & - & - & - & - & - & 2 & 1 & - & - & - & - \\
\hline & $\mathrm{I} 2 \mathrm{~B}$ & - & 1 & - & 3 & - & 2 & - & - & 1 & - & - \\
\hline & $\mathrm{I} 3 \mathrm{~A}$ & - & 4 & 1 & 1 & - & 13 & 1 & 6 & - & - & - \\
\hline \multirow[t]{4}{*}{ peine } & $\mathrm{I} 4 \mathrm{~A}$ & - & - & - & - & - & - & 2 & - & 6 & - & - \\
\hline & I4B & - & - & - & - & - & 1 & - & 1 & - & - & - \\
\hline & $\mathrm{I} 4 \mathrm{C}$ & - & - & - & - & - & - & 1 & - & - & - & - \\
\hline & $\mathrm{I} 4 \mathrm{D}$ & - & 1 & - & 1 & - & 11 & 1 & 1 & - & - & - \\
\hline \multirow[t]{9}{*}{ impresa } & $\mathrm{I} 5 \mathrm{~A}$ & - & - & - & - & - & 5 & - & - & 1 & - & - \\
\hline & $\mathrm{I} 5 \mathrm{~B}$ & - & - & - & - & - & 4 & 1 & 5 & 1 & - & - \\
\hline & $16 \mathrm{~A}$ & - & - & - & 1 & - & 6 & 1 & - & - & - & - \\
\hline & I6B & - & - & - & - & - & 1 & - & - & - & & - \\
\hline & I6C & - & - & - & - & - & 1 & - & - & - & - & - \\
\hline & $\mathrm{I} 7 \mathrm{~A}$ & - & - & - & - & 1 & - & - & - & - & - & - \\
\hline & $\mathrm{I} 9 \mathrm{~A}$ & - & 1 & - & 2 & 1 & 16 & 7 & 1 & 1 & - & - \\
\hline & I9 & - & 1 & - & - & - & - & - & - & - & - & - \\
\hline & I9C & - & - & - & - & - & 1 & - & - & - & - & - \\
\hline \multirow[t]{2}{*}{ acanalada } & $\mathrm{II} 1 \mathrm{~A}$ & - & - & - & 1 & - & 13 & - & 5 & - & - & - \\
\hline & II 1 в & 2 & 3 & 2 & 5 & - & 27 & 5 & 7 & 5 & - & - \\
\hline \multirow[t]{2}{*}{ incisa } & $\mathrm{II} 2 \mathrm{~A}$ & - & - & 1 & - & - & 6 & - & - & - & - & - \\
\hline & II $2 \mathrm{~B}$ & 1 & 1 & 1 & 2 & - & 15 & 1 & 2 & 5 & - & - \\
\hline \multirow[t]{2}{*}{ modelada } & III2 & - & 1 & 1 & - & - & 4 & 2 & - & 7 & - & - \\
\hline & III3 & - & - & 1 & 1 & - & - & 1 & - & 4 & - & - \\
\hline \multirow[t]{3}{*}{ Achakar } & $\mathrm{I} 9 \mathrm{E}$ & - & - & - & 2 & 1 & - & - & - & - & - & - \\
\hline & IV1 & 4 & - & 1 & 4 & - & - & - & - & - & - & - \\
\hline & Iv3 & - & - & - & 1 & - & - & - & - & - & - & - \\
\hline
\end{tabular}

FIG. 8. Desglose de técnicas decorativas por grupos principales, considerando UE y fase. 
aproximación, dicho grupo queda definido por el uso de conchas de bivalvos, normalmente estriadas, ya sean del género Pecten, Mimachlamys, Cerastoderma, Acanthocardia o similar, incluyendo algunos patélidos con costillas marcadas, observando técnicas de impresión o arrastre. Dentro de este grupo también hemos incluido aquellas impresiones realizadas aparentemente con valvas de conchas lisas, siendo más problemático en este sentido definir especies.

Así, las técnicas asociadas al arrastre cardial - I1 $\mathrm{F}$ - constituyen el tipo más extendido, con $48 \mathrm{ca}$ sos, 37 de forma individual (Fig. 8). Generalmente se aprecia bien el uso del dorso de la concha para peinar y cubrir presionando una amplia superficie del recipiente, bien formando haces continuos o bandas más o menos sencillas, diferenciándose pues de la forma de tratamiento conocida como arrastre cardial observada en el caso del País Valenciano (García, 2017). Otros 11 casos se hallan asociados a otras técnicas, mayoritariamente también cardiales al implicar el mismo instrumento, como impresiones verticales simples con concha dentada y cordón -I1A-III2, 1-, basculaciones con el filo de la concha en vertical -1 B, $2-$, impresiones sencillas con el filo de la concha en posición oblicua -I1C, 2-, así como asociadas a impresiones realizadas con instrumento indeterminado $-\mathrm{I} 9 \mathrm{~A}, 6-\mathrm{Al}$ arrastre cardial le siguen las impresiones sencillas con el filo de la valva en vertical-I1A, 8-, oblicuo-I1 C, 12-, basculante vertical -I1B, 7-y basculante oblicuo -I1E, 5-; impresiones sencillas oblicuas superpuestas -IDD, 5-, así como asociadas a cordones, impresiones simples y oblicuas -I1A, I1C-, con uno por cada caso (Fig. 9, n. ${ }^{\text {os }} 10-11$ y $\left.14-18\right)$.

En cuanto a las impresiones con concha no estriada o lisa, en algunos casos han sido difíciles de determinar debido a su propia morfología. Dentro de este grupo, situamos las impresiones verticales simples - I2A, 3- y basculantes verticales - Rocker, I2B, 3-. En el primer caso destaca particularmente un fragmento decorado a base de franjas formando un motivo espigado, aparentemente realizado con el filo de dos valvas entreabiertas de un mismo bivalvo de concha lisa -UE 1204 (Fig. 10, n. ${ }^{\circ}$ 7)-, hipótesis apoyada experimentalmente.

Tras el grupo de las cardiales, sin duda el grupo dotado de una mejor representación -72 fragmentos- es la Channeled Ware o acanalada (Gilman, 1975), denominada así al presentar acanaladuras o incisiones anchas comportando excisión de pasta, aparentemente realizadas con el canal sifonal de una cypraea, a juzgar por las impresiones en forma de coma con las que se asocian (Martínez et al., 2017). En este sentido, contamos con piezas que muestran acanaladuras largas y cortas, normalmente con el borde del canal sifonal, aunque también contamos con haces dobles, realizados con los dos bordes de dicho canal (Figs. 11-12). Así, las acanaladuras sencillas $-\mathrm{II} 1 \mathrm{~A}, \mathrm{II} 1 \mathrm{~B}-$ ascienden a 49 ejemplos, mientras que las piezas con impresiones de comas $-\mathrm{I} 3 \mathrm{~A}-$ se reducen a 9. Combinando ambas técnicas -impresión de comas y acanaladuras II $1 \mathrm{~A} / \mathrm{II} 1$ в у I $3 \mathrm{~A}-$, se presentan 10 ejemplares. Como prueba de su coincidencia cronológica, la asociación de alguna de estas técnicas propias de la Channeled Ware puede observarse con las propias del grupo Cardial como impresiones simples oblicuas -I1C, 1-, impresiones lineales oblicuas $-\mathrm{I} 5 \mathrm{~B}, 1-$, impresiones con instrumento no determinado $-\mathrm{I} 9 \mathrm{E}-\mathrm{o}$ incisiones $-\mathrm{II} 2 \mathrm{~B}$, $1-$. En este sentido, un número mayor de estas asociaciones pueden observarse entre las cerámicas procedentes de excavaciones anteriores ${ }^{6}$.

Las cerámicas decoradas a peine suponen un grupo muy característico, con 24 fragmentos contabilizados en esta fase. La técnica más frecuente es la impresión basculante-I4D, 13-. Aquí, el aspecto de la trama decorativa cambia considerablemente en función de la posición inclinada o vertical del instrumento. En este sentido contamos con motivos abigarrados que pueden ser confundidos con secuencias de boquique y sillon d'impression (Alday y Moral, 2011), o bien con roulettes o matrices de rueda dentada (Jórdeczka et al., 2011), si bien la experimentación desarrollada apunta claramente a matrices dentadas basculantes para la ejecución de estos motivos. Algunos de ellos serían realizados con

6 El Idrissi, op. cit. n. 2. 

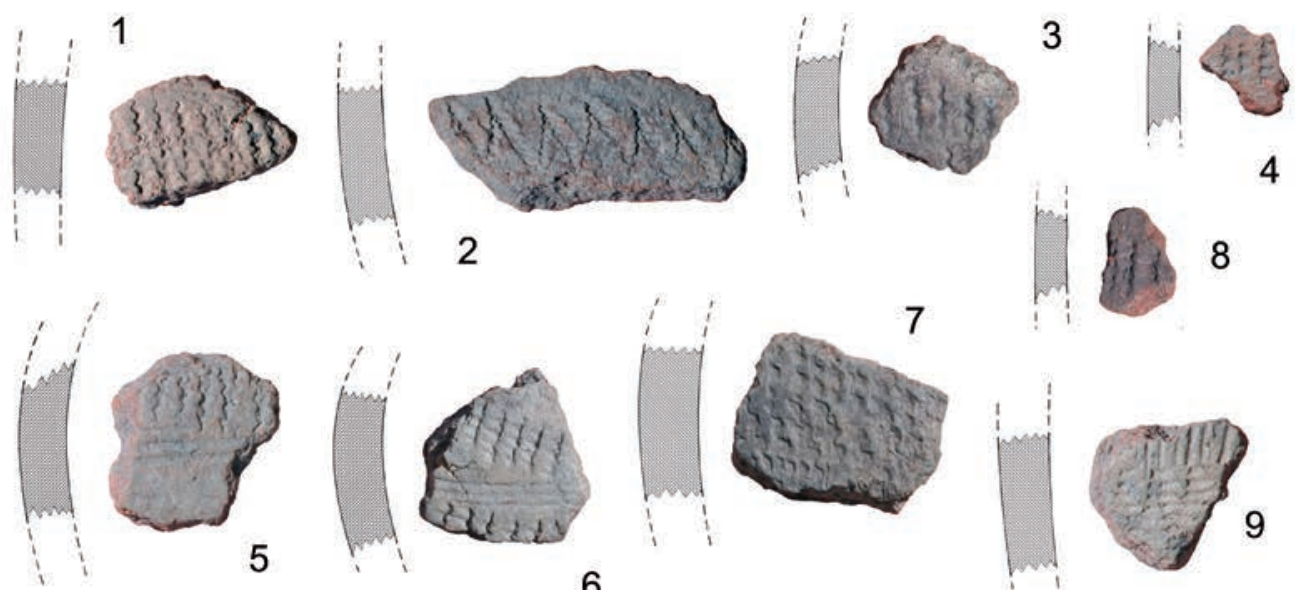

4

$5 \quad 6$
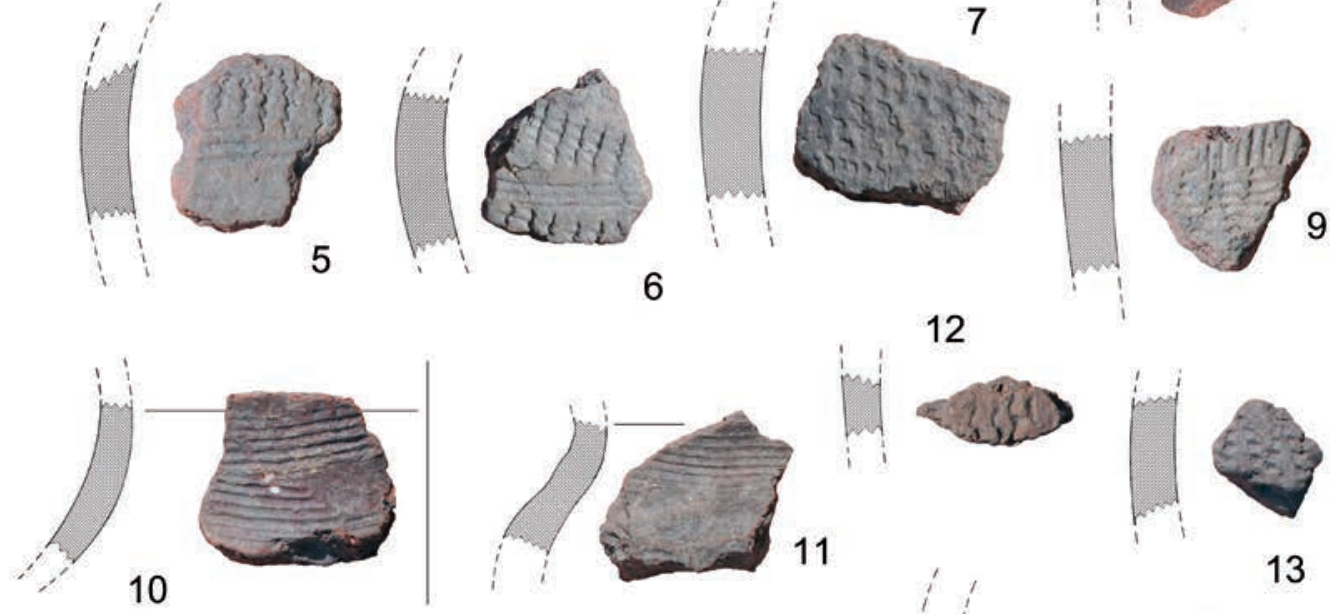

12
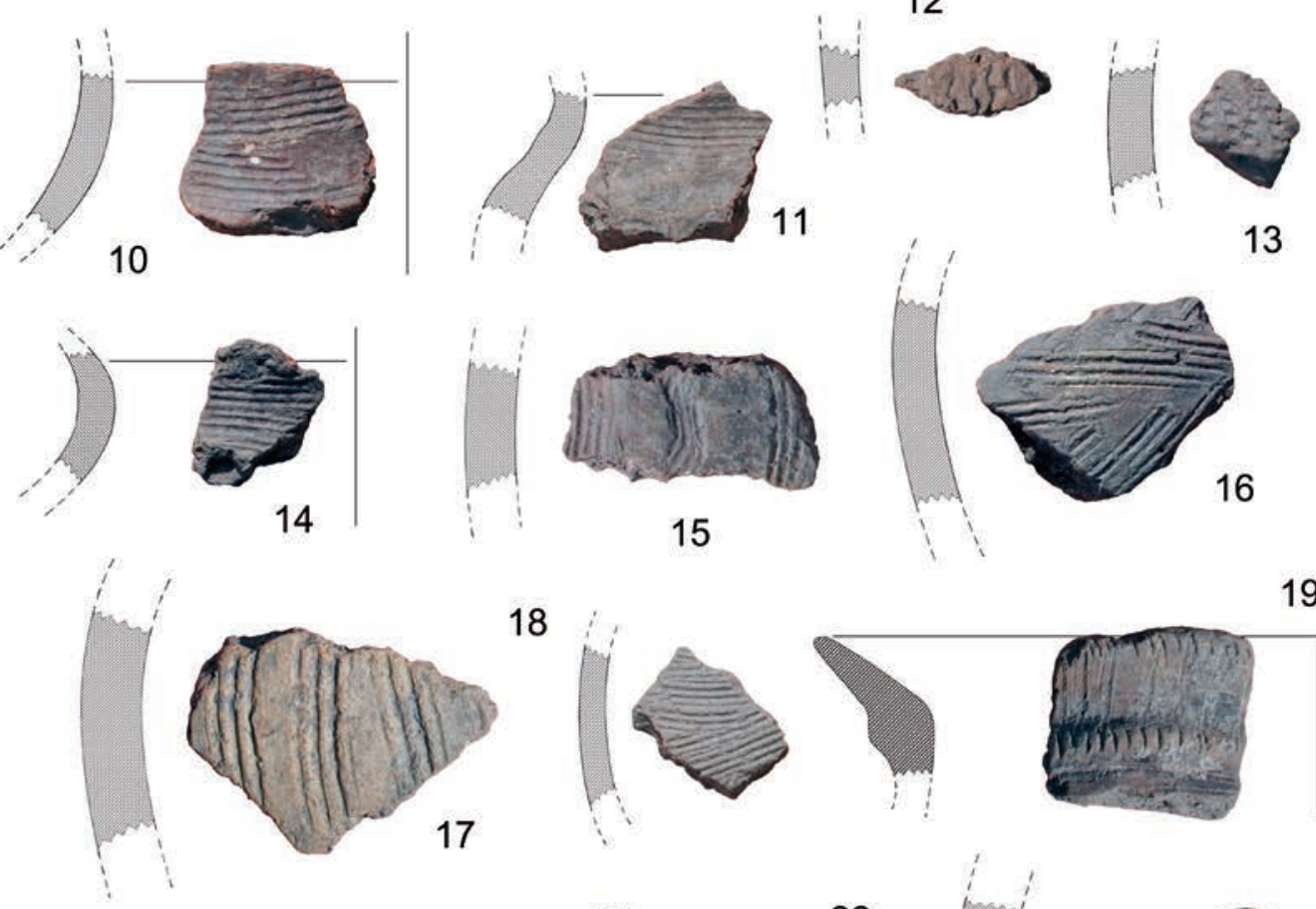

18

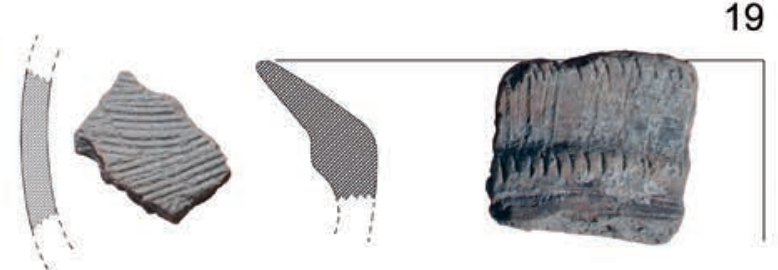

19
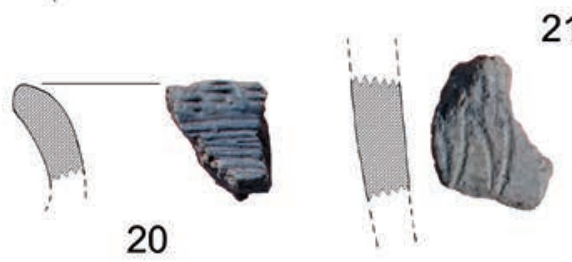

21

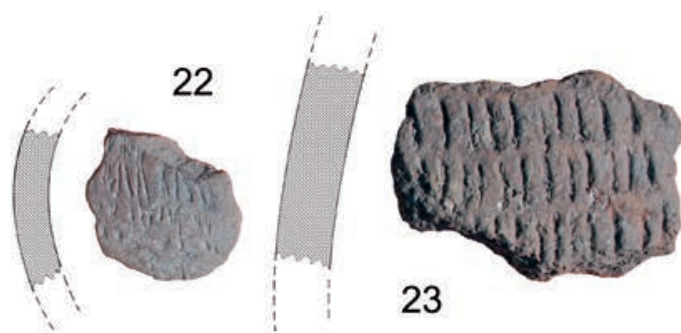

$20 \mathrm{~cm}$

FIG. 9. Fragmentos decorados del Neolítico Antiguo procedentes de la UE 1103-1: 1-9 y 12-13) impresión cardial; 10, 11, 1418, 20) peine (arrastre) cardial; 19) impresión de concha levemente estriada (¿Patella?) asociada a arrastre; 21 y 23) impresión con concha lisa; 22) impresión pivotante (¿Patella?). 
46 R. M. Martínez Sánchez, J. C. Vera Rodríguez, G. Pérez Jordà y Leonor Peña-Chocarro / La decoración cerámica en el Neolítico...
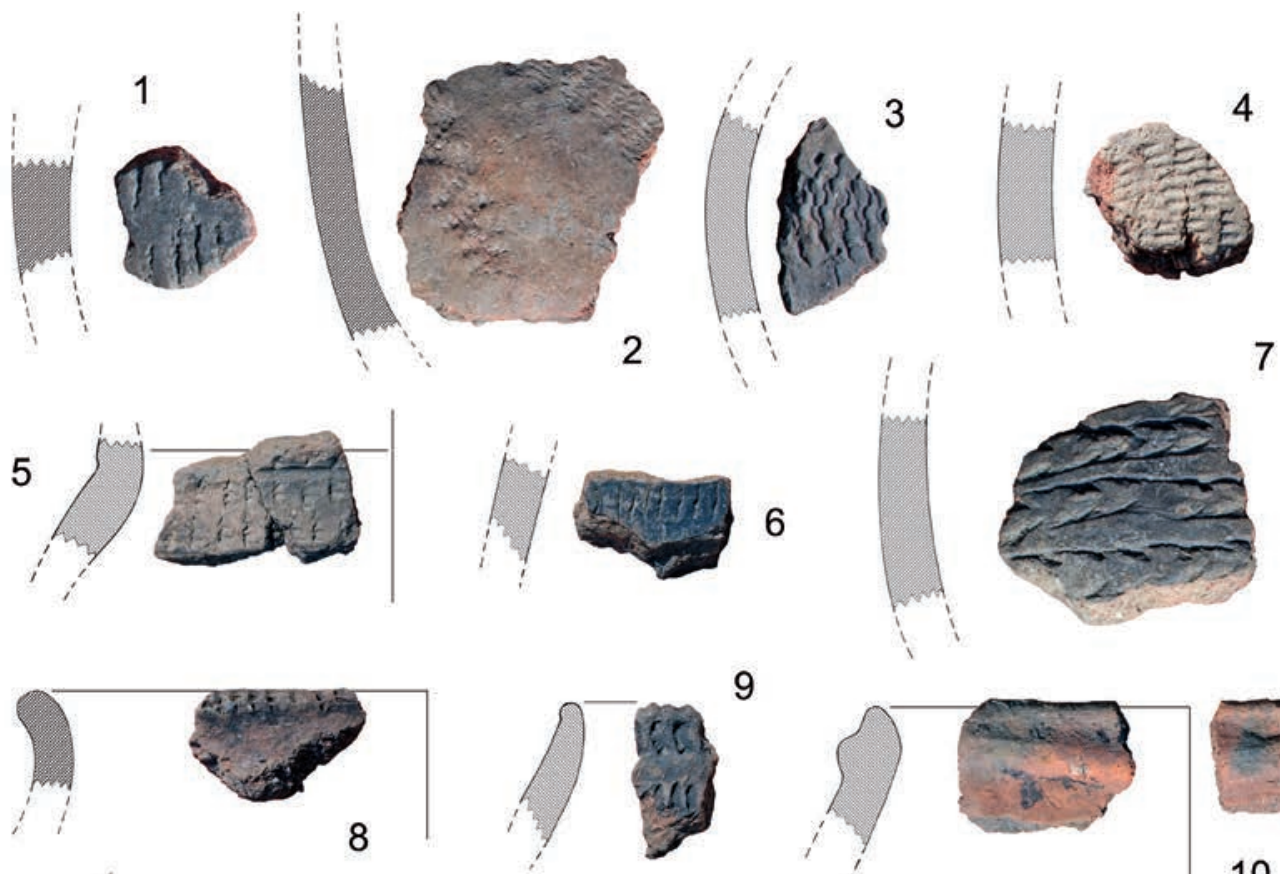

9
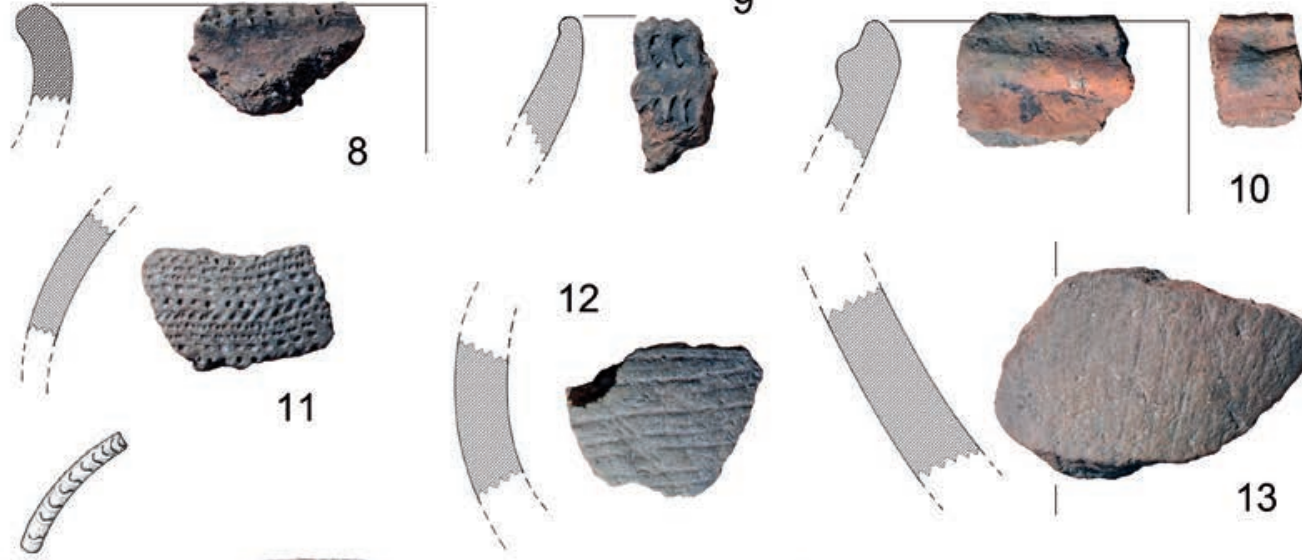

11
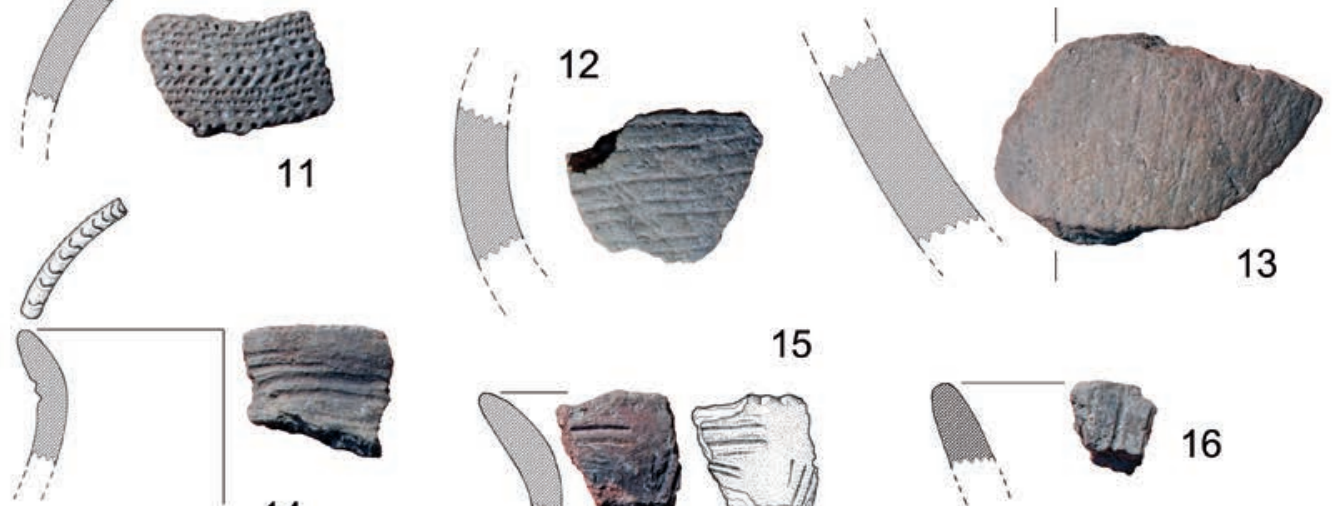

14
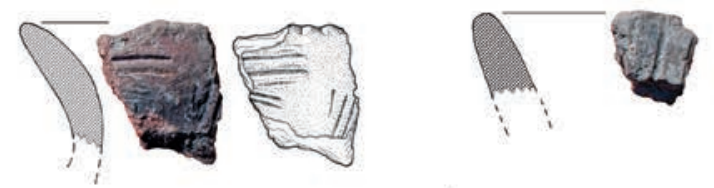

16
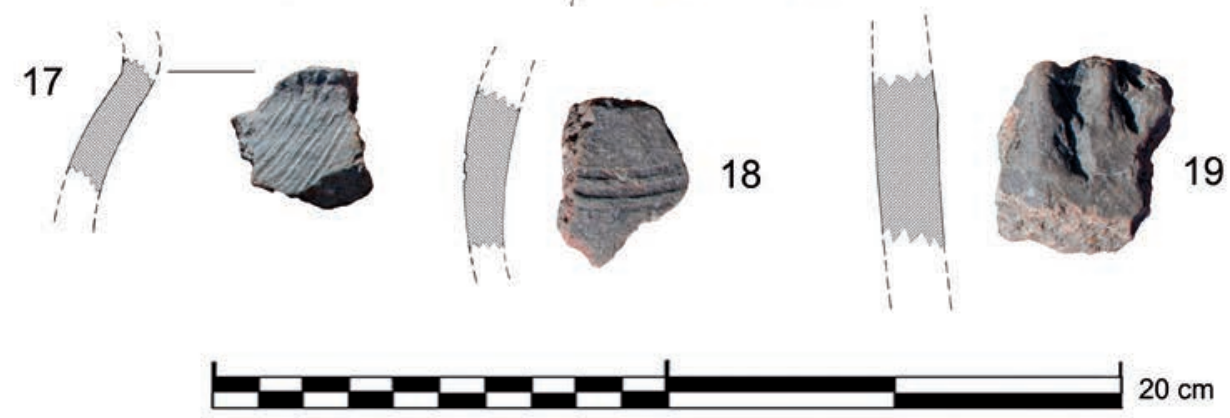

Fig. 10. Fragmentos cerámicos del Neolitico Antiguo procedentes de la UE 1204-1: 1-6) impresión cardial (Acanthocardia 3 y Patella 6); 7) impresión doble con concha lisa (valvas entreabiertas); 8, 9 y 11) impresión a peine y matriz dentada curva; 10) cordón aplicado; 11) acanaladas asociadas a impresiones de comas; 12-14) arrastre doble del plano ventral del canal sifonal de cypraea; 18) peinado cardial; 19) impresiones oblicuas superpuestas con instrumento indeterminado. 

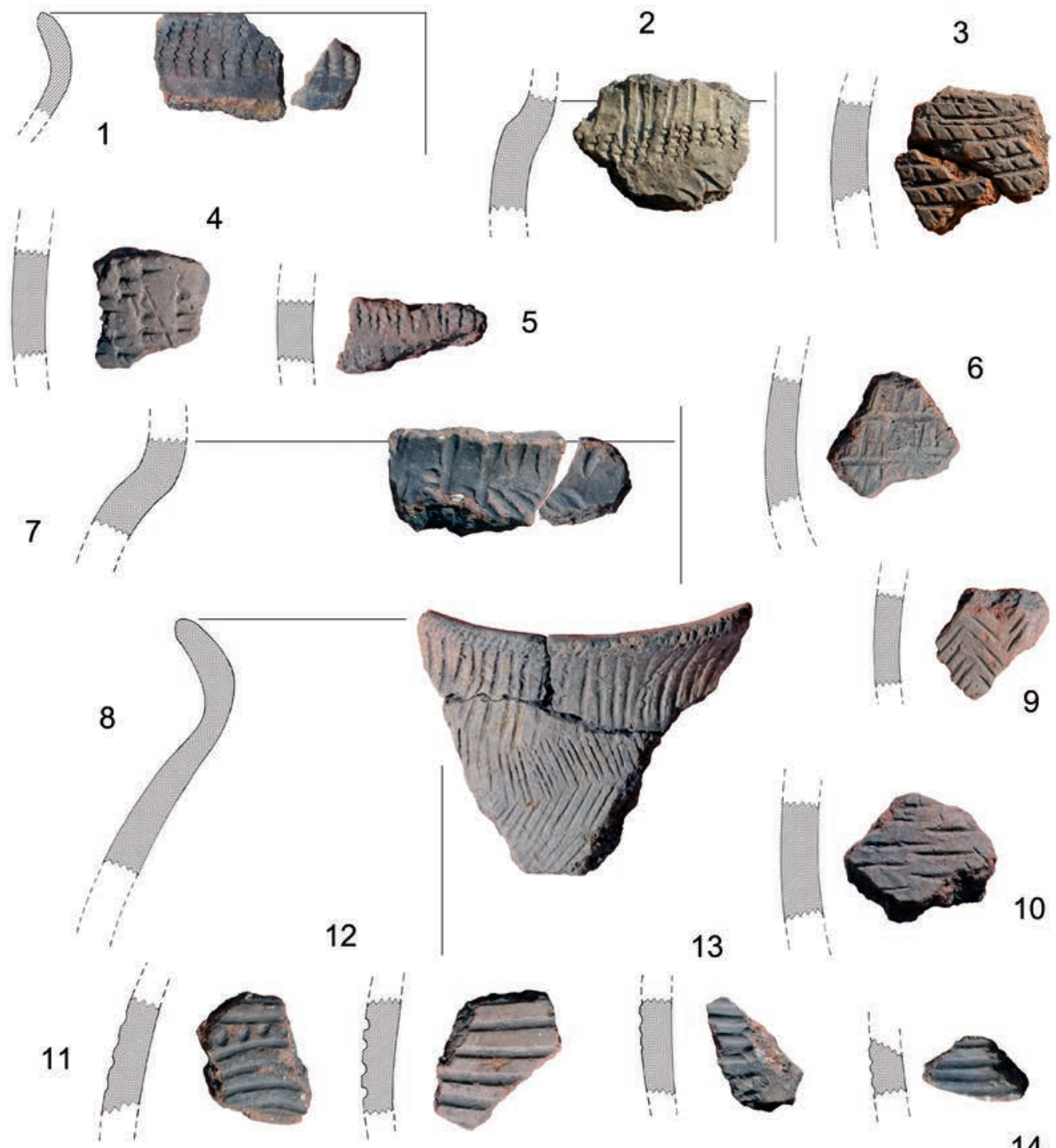

12

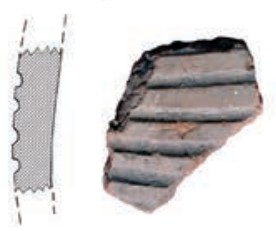

15
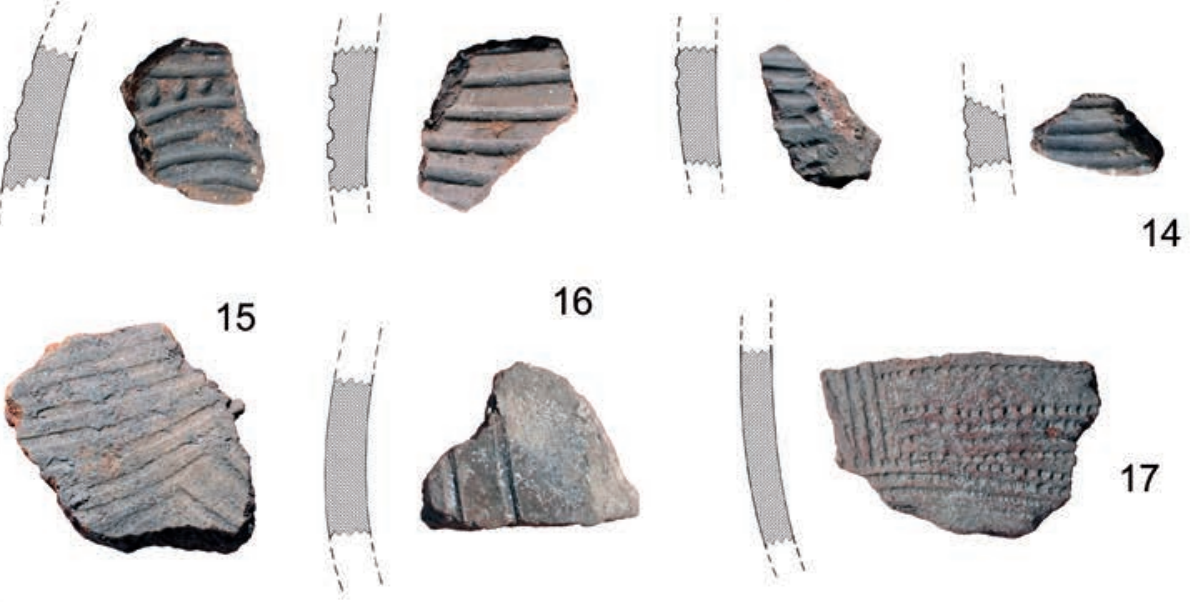

14
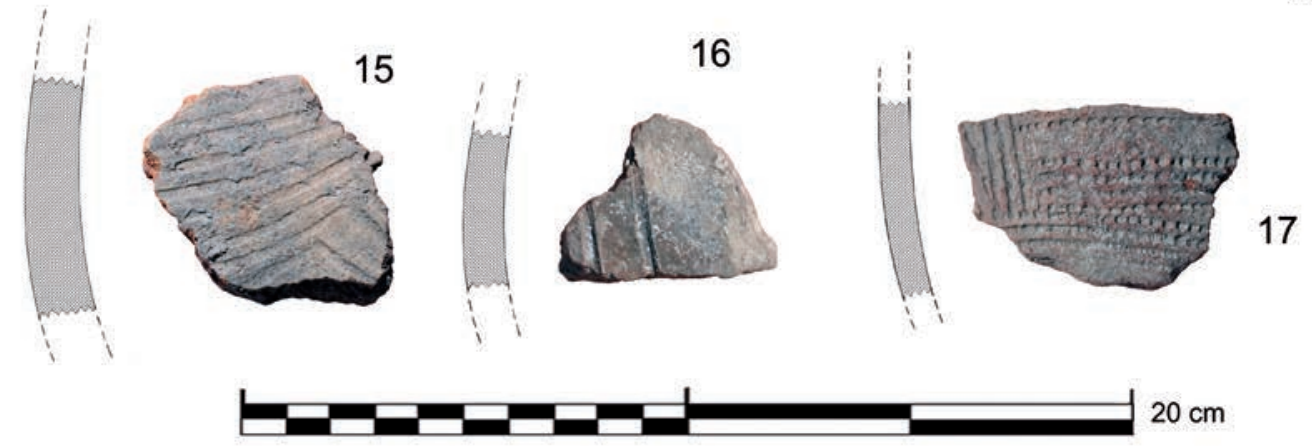

Fig. 11. Fragmentos decorados del Neolitico Antiguo procedentes de las UUEE 1003 (1), 1207 (2), 1104 (3) y 1103-2 (4-17): 1, 2, 4 y 5) impresión cardial; 3 y 6) incisas; 8-10) impresas; 7-8 y 11-16) acanaladas (y en asociación a impresiones de comas); 17) impresión a peine. 
48 R. M. Martínez Sánchez, J. C. Vera Rodríguez, G. Pérez Jordà y Leonor Peña-Chocarro / La decoración cerámica en el Neolítico...
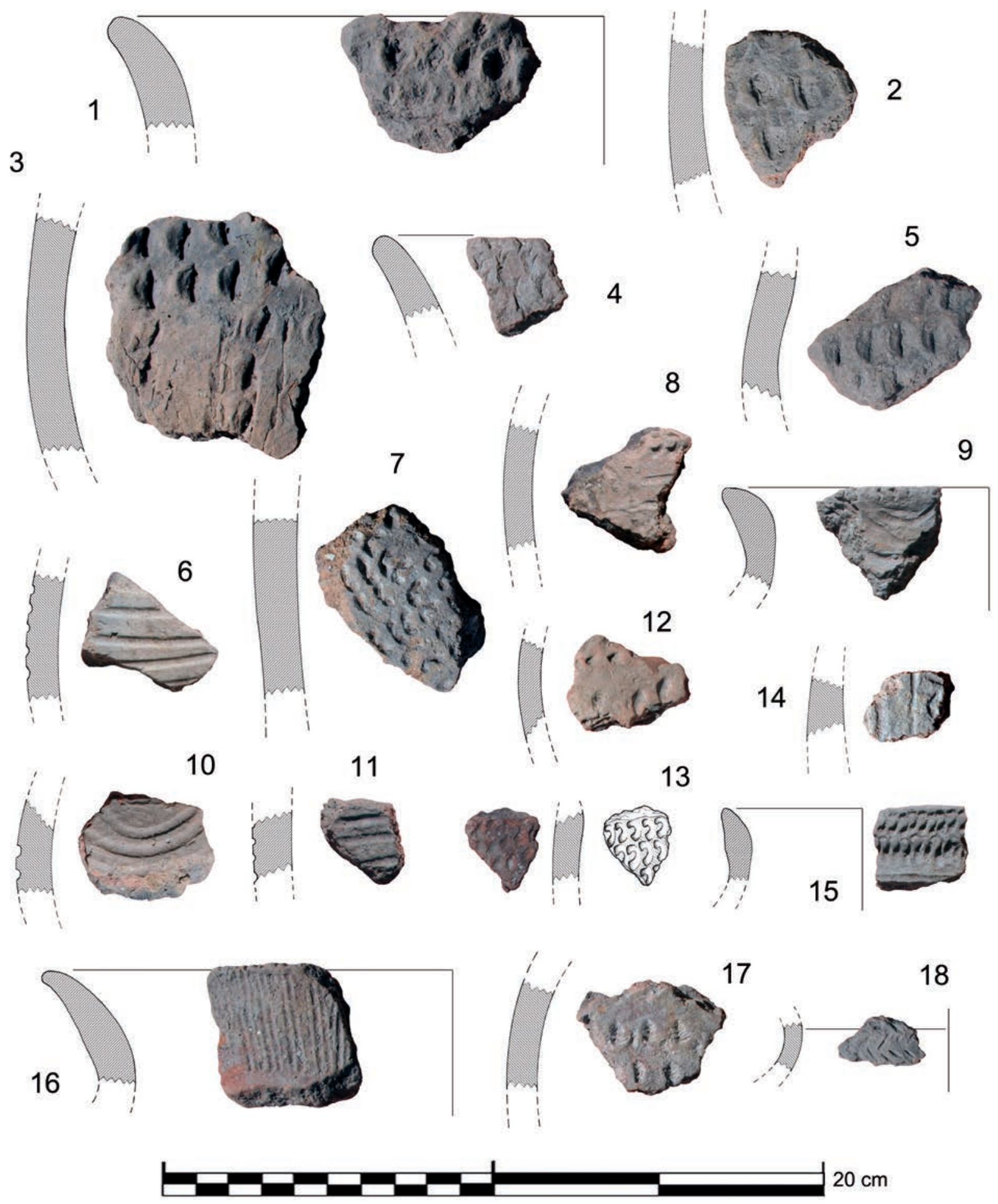

FIG. 12. Fragmentos decorados del Neolítico Antiguo procedentes de la UE 1103-1: 1-5) impresas digitadas; 6-16) acanaladas asociadas a impresiones de comas; 17-18) impresas (vertical y oblicua lineal). 
cierto ritmo e inclinación de la matriz dentada, y otros ejecutados verticalmente, resultando motivos muy diversos según la forma y el uso del instrumento. Otros motivos ejecutados claramente con estas matrices han sido clasificados como impresiones simples verticales $-\mathrm{I} 4 \mathrm{~A}, 4-$, oblicuas $-\mathrm{I} 4 \mathrm{C}, 1-$, impresiones sencillas superpuestas, formando carreras -I4B, 2-, acompañados de asociaciones, bien de cordones $-\mathrm{I} 4 \mathrm{~A} / \mathrm{III} 2$, 3-, o incisiones $-\mathrm{I} 4 \mathrm{~A} / \mathrm{II} 2 \mathrm{~B}, 1-$ (Fig. 13).

Como grupo independiente no adscribible a los anteriores, hemos definido el de las impresiones variadas con instrumento indeterminado, con hasta 50 elementos, incluyendo fragmentos que se asocian con decoraciones presentes en otros grupos. En este hemos incluido aquellos que muestran impresiones realizadas por matrices diversas, punzones, espátulas y objetos indeterminados, así como con los dedos -digitada, pellizcada o ungulada-. Dentro de este grupo destacan las impresiones verticales $-\mathrm{I} 5 \mathrm{~A}$, $5-$, en las que se observan instrumentos apuntados y, en algún caso, elementos naturales como los nódulos de ciertas especies de gasterópodos marinos -¿Hexaplex, Ocenebra?- para realizar impresiones

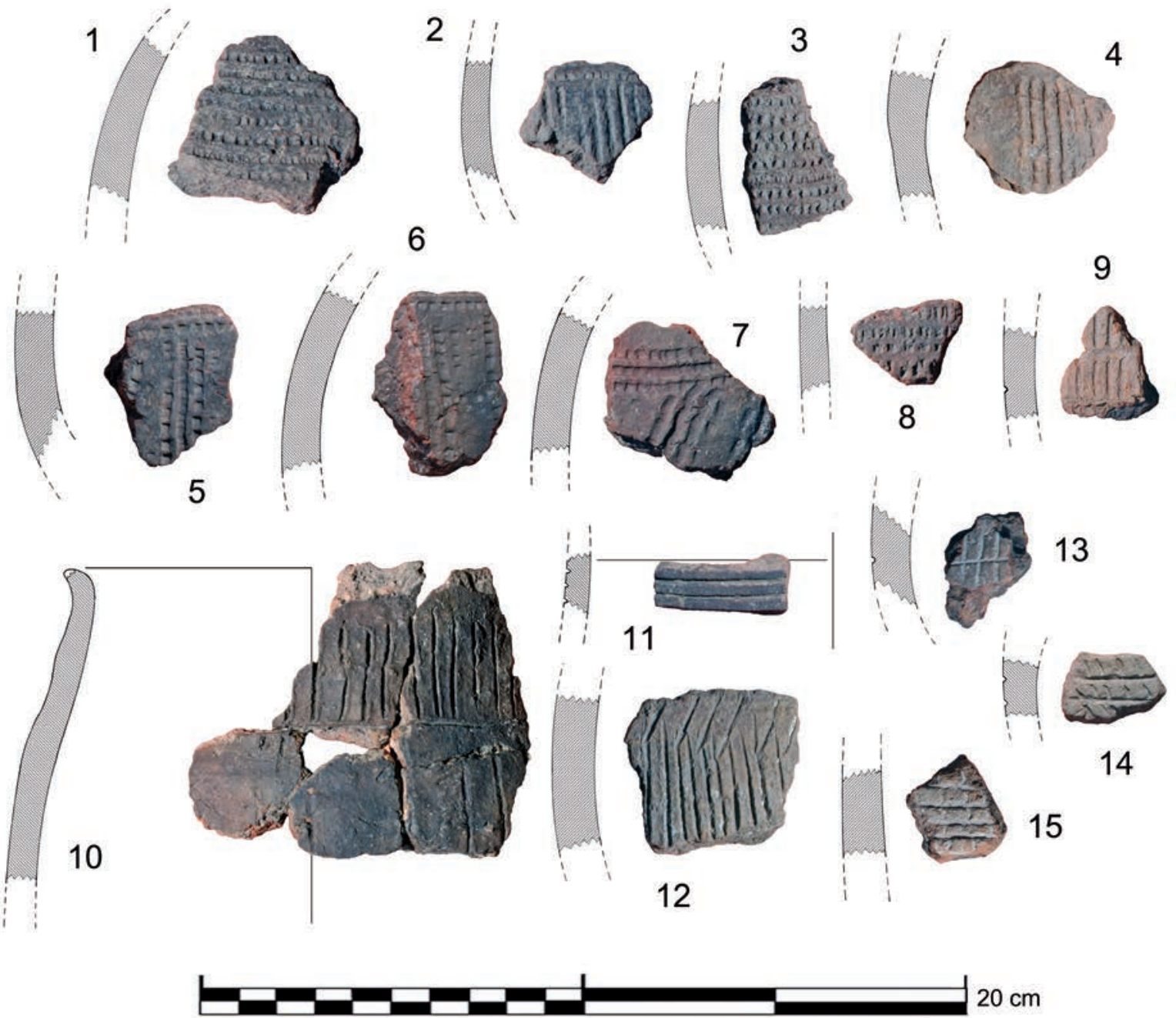

FIG. 13. Fragmentos decorados del Neolitico Antiguo procedentes de la UE 1103: 1-8) impresas a peine; 9-11 y 13-15) incisas; 12) impresión oblicua lineal. 
(Fig. 12, n. ${ }^{\circ}$ 17). Más significativas resultan las impresiones oblicuas - con frecuencia incluidas dentro de las incisas-, realizadas con instrumento de punta aguda $-15 \mathrm{~B}, 7-$, dispuestas a menudo formando motivos en zigzag-Herringbone- (Fig. 11, n. ${ }^{\text {os } 8-9) . ~}$ Otras son aquellas realizadas con la punta de los dedos, las cuales se han dividido en ungulaciones $-\mathrm{I} 6 \mathrm{~A}, 5-$, pinzado-I6B-e impresiones digitales -I6C, $1-$, con asociaciones a impresiones indeterminadas $-\mathrm{I} 6 \mathrm{~A} / \mathrm{I} 9 \mathrm{~A}, 1-\mathrm{o}$ entre dos técnicas digitales $-\mathrm{I} 6 \mathrm{~A} / \mathrm{I} 6 \mathrm{~B}$, 1 - (Fig. 12, n. ${ }^{\text {os }} 1-5$ ). Por último, se citan impresiones con instrumento indeterminado $-\mathrm{I} 9 \mathrm{~A}-$, las cuales aparecen aisladas en 16 fragmentos, asociadas a arrastre cardial $-\mathrm{I} 1 \mathrm{~F} / \mathrm{I} 9 \mathrm{~A}, 6-$, a ungulaciones $-\mathrm{I} 6 \mathrm{~A} /$ I9A, 1- o a incisiones - II $2 \mathrm{~B}, \mathrm{I} 9 \mathrm{~A}, 2-$. Las impresiones oblicuas superpuestas con instrumento indeterminado $-\mathrm{I} 9 \mathrm{C}-$ se reducen a un solo caso (Fig. 10, n. $\left.{ }^{\circ} 19\right)$.

Otro grupo importante en la fase antigua es el de la cerámica modelada o con añadidos plásticos, con 18 fragmentos. Como viene siendo habitual en otros sitios del Neolítico Antiguo de la Península Tingitana, los EPS y dispositivos tipo asa, aunque conocidos, no resultan demasiado frecuentes. En nuestro caso tan solo hemos identificado un mamelón perforado. Dentro de los añadidos plásticos, son los cordones -III2- los más representados. Aparecen de forma aislada en 7 casos, y asociada a otras técnicas, a menudo sobre el cordón o bajo este, a impresiones a peine verticales simples $-\mathrm{I} 4 \mathrm{~A} / \mathrm{III} 2$, $3-$, cardiales verticales $-\mathrm{I} 1 \mathrm{~A} / \mathrm{III} 2,1-$, a impresiones cardiales verticales y arrastre cardial $-\mathrm{I} 1 \mathrm{~A} / \mathrm{I} 1 \mathrm{~F} / \mathrm{III} 2$, $1-\mathrm{o}$ a incisiones $-\mathrm{I} 2 \mathrm{~A} / \mathrm{I} 2 \mathrm{~B} / \mathrm{III} 2,1-$. Otros añadidos plásticos -refuerzos, pastillas o mamelones sencillos (III3, 4) - tan solo parecen asociados a impresiones oblicuas cardiales -I1c/III3, 1- (Fig. 14, n. ${ }^{\circ} 13 \mathrm{y}$ Fig. 15).

Por último, las piezas con incisiones -26- se agrupan en cortas -II $2 \mathrm{~A}, 4-$, asociadas a otras incisiones largas $-\mathrm{II} 2 \mathrm{~A} / \mathrm{II} 2 \mathrm{~B}, 1-$, a cordones $-\mathrm{II} 2 \mathrm{~A} /$ II 2 в/III3, 1-, у a su vez en largas -II2B, 13-, que se asocian a su vez a impresiones simples verticales a peine $-\mathrm{I} 4 \mathrm{~A} / \mathrm{II} 2 \mathrm{~B}, 1-$, impresiones verticales con instrumento apuntado $-\mathrm{I} 5 \mathrm{~A} / \mathrm{I} 2 \mathrm{~B}, 1-\mathrm{o}$ impresiones oblicuas de líneas $-\mathrm{I} 5 \mathrm{~B} / \mathrm{I} 2 \mathrm{~B}, 3-$. Un motivo

Ediciones Universidad de Salamanca / @®@০ relativamente frecuente asociado a esta técnica es el de las incisiones perpendiculares formando una trama en damero -quadrillé-, presente desde el inicio de la secuencia (Fig. 11, n.o 3).

Como ya adelantamos, las formas documentadas prácticamente se reducen a formas cerradas; divididas a su vez en formas globulares con labio diferenciado exvasado y cuello estrangulado, de entre 110 y $200 \mathrm{~mm}$ de diámetro, de cierta profundidad y aspecto anfórico, y vasos globulares de labio indiferenciado y borde entrante, de proporciones similares. Los microvasos estarían presentes ${ }^{7}$, si bien en nuestro caso solo han podido determinarse en conjuntos superficiales o descontextualizados, con decoraciones incisas -a quadrillée o impresiones cardiales.

\subsubsection{La cerámica del Neolítico Medio}

Este conjunto resulta numéricamente inferior al de la fase anterior. Así, en total la suma de fragmentos asciende a 224 , con la problemática particular que supone identificar una gran parte de este conjunto como elementos en origen pertenecientes al Neolítico Antiguo y, por tanto, residuales. En ese sentido, la sola existencia de una datación radiocarbónica en estos niveles, adjudicada a la segunda mitad del v milenio cal AC -Beta-331986, $5400 \pm$ 30 вр-, reforzaría la existencia de ocupaciones humanas propias del Neolítico Medio, incorporando igualmente materiales anteriores. El reconocimiento de cerámicas identificadas dentro del grupo de las Ashakar Ware (Gilman, 1975; Martínez et al., 2018a), y su concordancia temporal con la data obtenida, permite cierta confianza para atribuir al menos dicho grupo cerámico a la segunda mitad del v milenio cal AC (Fig. 16).

A diferencia del conjunto anterior, las cerámicas de esta fase muestran una clara proporción en favor de las no decoradas; 160 frente a 64 de las decoradas, $71 \%$ frente a un $29 \%$. Ello resulta significativo, al incluir entre las decoradas aquellas dotadas de engobes de color, como veremos, rojo o beige.

7 El Idrissi, op. cit. n. 2. 

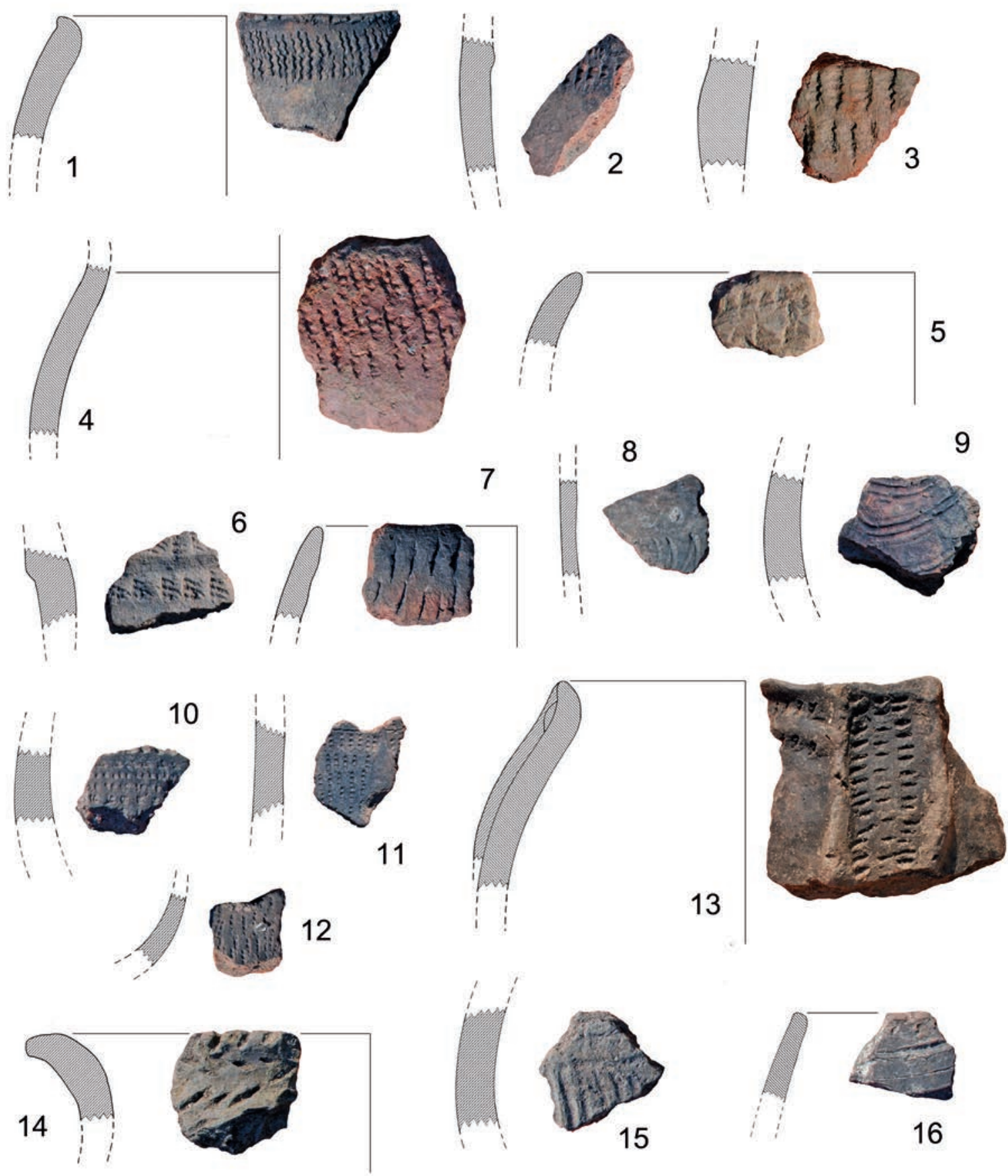

$20 \mathrm{~cm}$

FIG. 14. Fragmentos decorados procedentes de la UE 1204-2 datados en el Neolitico Antiguo: 1-6) impresiones cardiales; 7) de posible Patella; 8) impresión basculante de concha lisa (rocker); 9) peinado cardial (arrastre); 10-13) impresión a peine o instrumento de puntas múltiples (el 13 asociado a cordones); 14) impresión oblicua lineal; 15) acanalada; 16) incisa. 
52 R. M. Martínez Sánchez, J. C. Vera Rodríguez, G. Pérez Jordà y Leonor Peña-Chocarro / La decoración cerámica en el Neolitico...
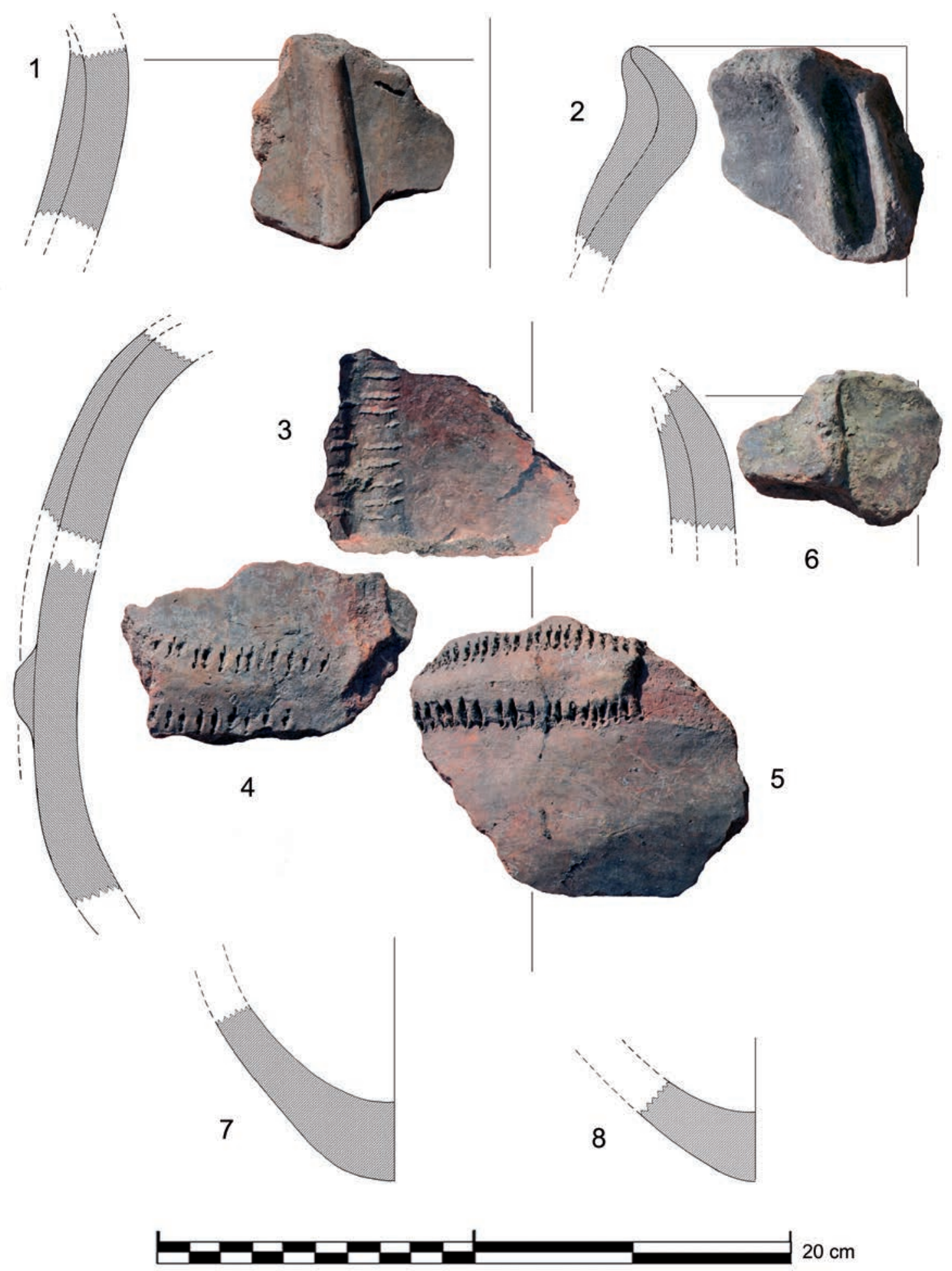

FIG. 15. Fragmentos decorados del Neolitico Antiguo procedentes de la UE 1204-2: 1-5) fragmentos con cordones aplicados; 3-5) cordones asociados a impresiones de matriz dentada. 

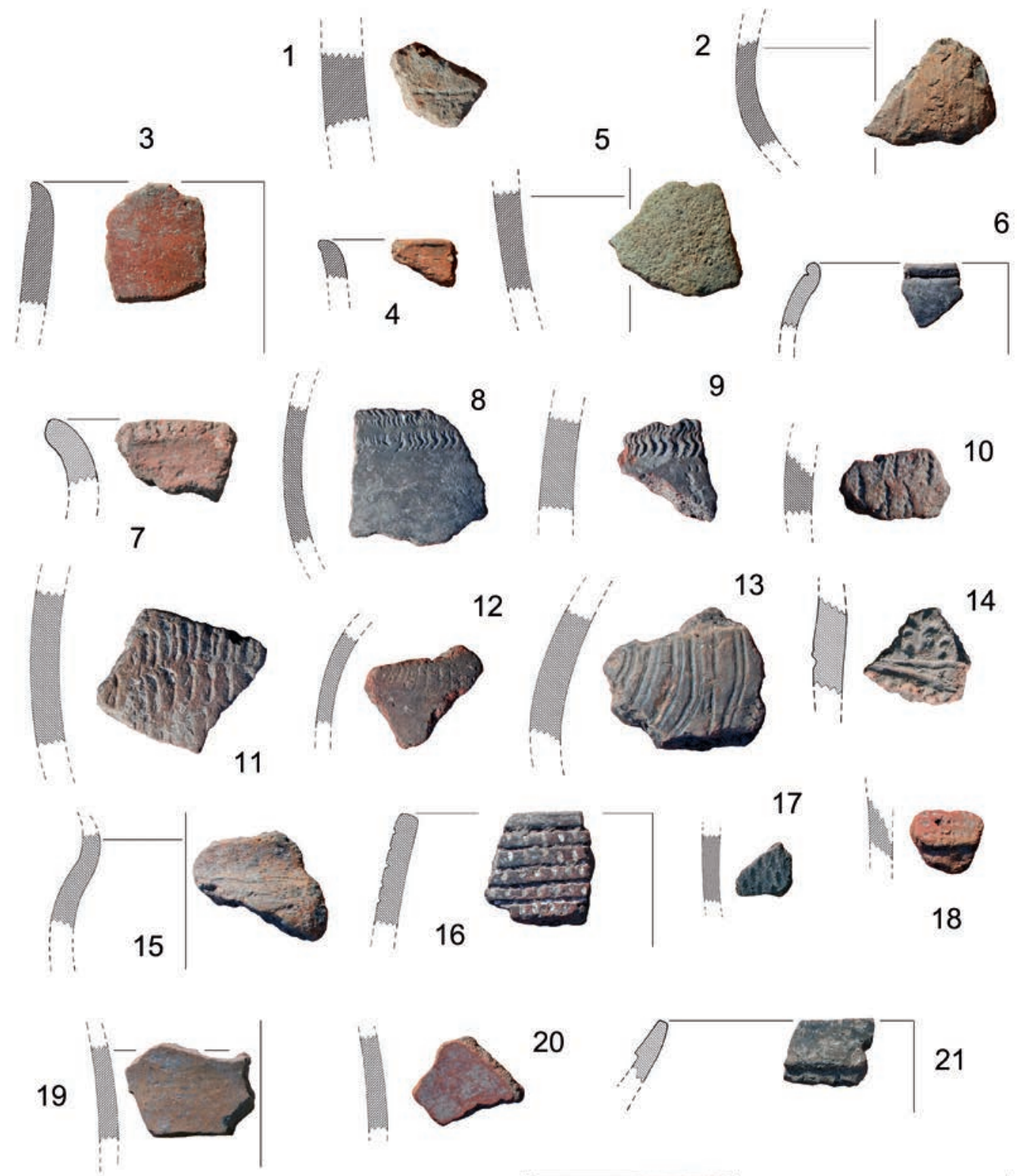

\section{1}
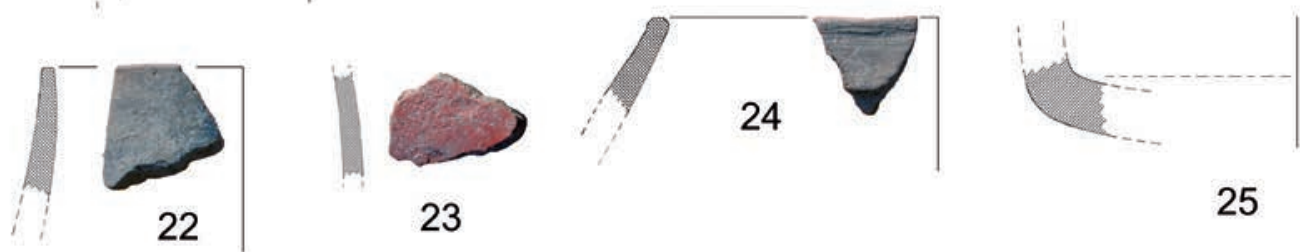

23

Fig. 16. Fragmentos cerámicos del Neolítico Medio procedentes de las UUEE 1101-3 (1-6) y 1102 (7-25): 3-6, 16-17 y 19-25) grupo Achakar; 7-10) impresión cardial (8 y 9 Pecten); 11-12) impresiones con concha lisa; 2 y 13-14); 1 y 15) incisas; 18) impresión a peine. 
Comenzando con el grupo de las impresiones cardiales, estas se componen de 22 fragmentos, incluyendo asociaciones con otros grupos técnicos y aparentemente mostrando cierta tendencia a la diversidad en las especies empleadas -Acanthocardia, Pecten-. Como en la fase anterior, destaca en primer lugar el arrastre cardial -I1F, 7-, si bien en mucha menor proporción. Muy por detrás quedan otras técnicas, como la impresión basculante con filo vertical -I1 в, 5-, las impresiones simples verticales -I1A, 3-, siguiendo con las impresiones simples con concha oblicua -I1c, 1-, la combinación de esta con las simples verticales $-\mathrm{I} 1 \mathrm{~A} / \mathrm{I} 1 \mathrm{C}, 1-$, la asociación del peinado cardial con cordones $-\mathrm{I} 1 \mathrm{~F} / \mathrm{III} 2$, $1-$. Por último, existe una relativamente aceptable representación de basculantes verticales con concha lisa, -I2B, 4-, donde podrían observarse valvas de navaja entre otras especies.

La Channeled Ware también se encuentra presente en esta fase con 16 fragmentos, si bien, como el caso del grupo anterior, deben ser interpretados como elementos erráticos procedentes de la fase infrayacente. Así, contamos con impresiones verticales de comas $-\mathrm{I} 3 \mathrm{~A}, 4-\mathrm{y}$ la asociación de estas con acanaladuras largas $-\mathrm{I} 3 \mathrm{~A} / \mathrm{II} 1 \mathrm{~B}, 2-$, acanaladuras cortas sin asociación -II 1A, 1-, largas -II1B, 7-, asociaciones entre acanaladuras e incisiones $-\mathrm{II} 1 \mathrm{~B} / \mathrm{II} 2 \mathrm{~A}$, $1 ;$ ІІ2в/ІІ1 1 , $1-$.

Las cerámicas a peine son poco significativas, apenas dos identificadas como peine basculante -I4D-, compatibles con elementos residuales. Las impresas no cardiales -7- cuentan con ejemplos de digitaciones-I6A, 1-, impresiones curvas no determinadas $-\mathrm{I} 7 \mathrm{~A}, 1-$, impresiones con útil no determinado $-\mathrm{I} 9 \mathrm{~A}$, 4- o impresiones basculantes - $\mathrm{I} 9 \mathrm{~B}, 1-$. Las modeladas -4- cuentan con un ejemplo de asociación cardial arrastrado y cordón -I1F/III2, 1-, impresiones verticales sencillas cardiales y cordón $-\mathrm{I} 1 \mathrm{~A} / \mathrm{III} 2$, $1-\mathrm{y}$ dos fragmentos con cordones individuales III2, 2-. Para terminar, las incisas -6- constan de las asociadas a acanaladas ya nombradas $-\mathrm{II} 1 \mathrm{~B} / \mathrm{II} 2 \mathrm{~A}$, 1; ІІ2 $\mathrm{B} / \mathrm{II} 1 \mathrm{~B}, 1-$, así como incisiones largas presentes en 4 piezas -II2B, 4- (Fig. 17).

Para terminar, el verdadero punto diferenciador de esta fase es la presencia de técnicas decorativas -así como formas- absolutamente novedosas y no presentes en la fase anterior. Esta serie de caracteres se agrupan en la conocida como Ashakar Ware, un grupo de cerámicas identificadas en los yacimientos del área de Tánger y caracterizadas por formas de paredes rectas, bases planas y asas en funicular, bordes reforzados y acanalados y decoraciones a roulette, con cuerda enrollada, a menudo formando motivos a barbed wire o cuentas de rosario. Será en este grupo donde se observará por primera vez el uso de engobes rojos o beiges, rematando acabados muy cuidados. En este sentido, los fragmentos decorados que hemos atribuido a este grupo son 12 , junto a 6 no decorados, pero de rasgos y forma compatibles con este grupo. Así y comenzando por los engobes de color característicos de este grupo -Iv1- contamos con 10 fragmentos, dominando los tonos rojizos sobre los pardos o beiges. Contamos con dos casos claros de impresiones a roulette $-\mathrm{I} 9 \mathrm{E}$, $2-\mathrm{y}$ un interesante caso combinando acanaladuras, roulette e incrustaciones de pasta blanca $-\mathrm{I} 9 \mathrm{E}, \mathrm{II} 1 \mathrm{~B}$, Iv3, 1-. Junto a las propias de la fase anterior, y de cuyo origen residual ya hemos dado cuenta, las formas observadas en este conjunto se componen de vasos de paredes rectas, con perfiles troncocónicos y tubulares, con bordes no diferenciados, observándose un menor grosor de las paredes y un aspecto marcadamente diferente en cuanto a tecnología, con desgrasantes finos y aspecto cuidado. No hemos documentado, a diferencia de en El-Khil, asas funiculares (Martínez et al., 2018a).

\section{Discusión}

\subsection{Las cerámicas neoliticas de Kaf Taht el-Ghar y su temporalidad}

El análisis de las cerámicas de la fase neolítica de Kaf Taht el-Ghar pone de manifiesto la vigencia de las observaciones realizadas hace más de 40 años por A. Gilman respecto a los sitios del área tangerina. El grupo asociado a decoraciones cardiales de concha estriada, acanaladas y, en menor medida, a base de impresiones variadas y apliques plásticos, 

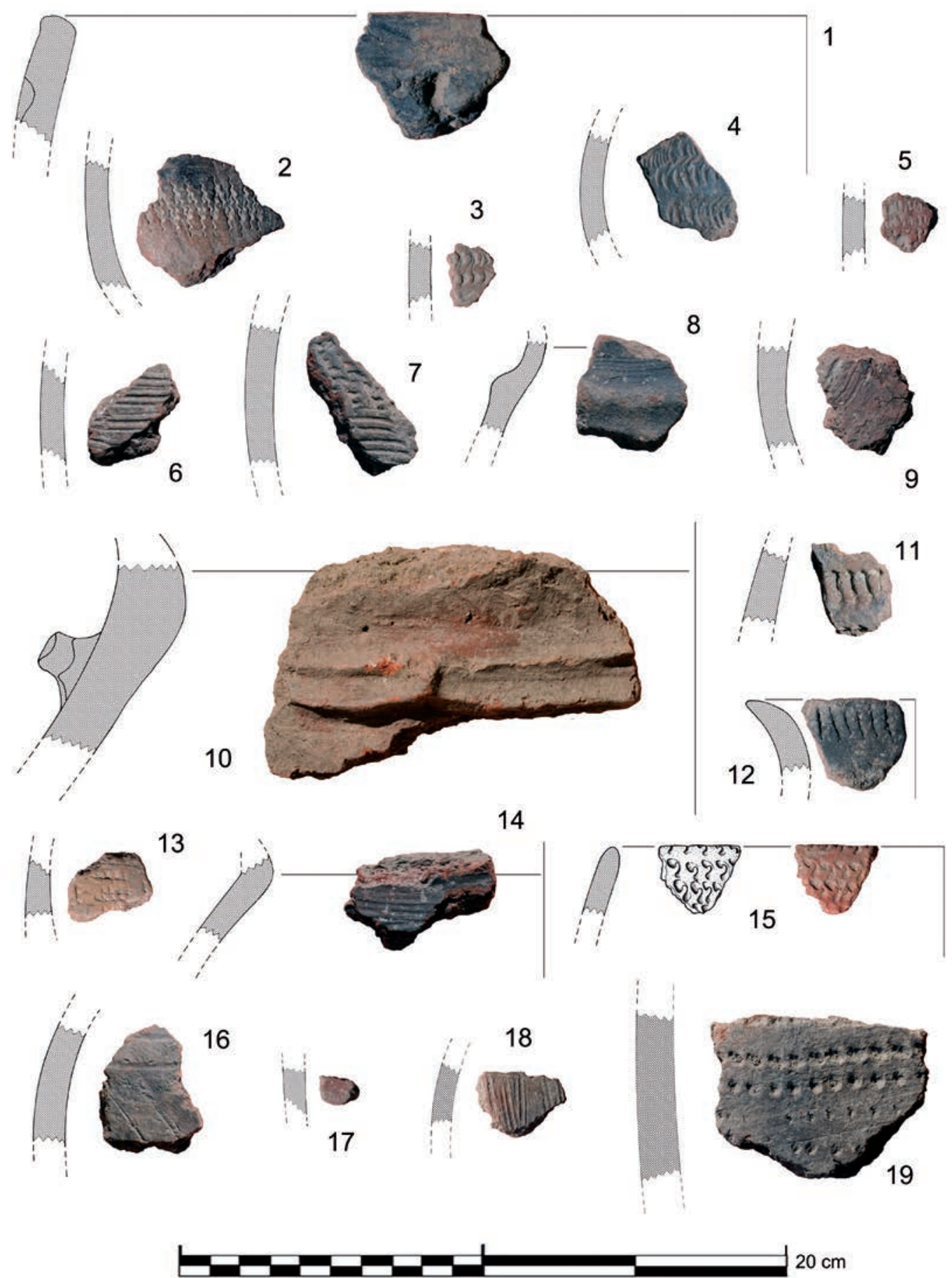

Fig. 17. Fragmentos cerámicos del Neolitico Medio procedentes de las UUEE 1202-1 (1-9), 1202-2 (10-17) y 1203 (18-19): 1) impresión indeterminada, posible digitación; 2-3 (Pecten), 12 (Patella) y 13) impresión cardial; 4) impresión basculante con concha lisa; 5) impresión basculante a peine; 6-7 y 15-16) grupo de las acanaladas; 8, 9, 14 y 18) peinado cardial; 10) cordones modelados; 11) impresiones con instrumento indeterminado;19) impresiones a roulette; 17 y 19) grupo Achakar. 
con frecuencia mostrando forma anfórica, base apuntada y cuello estrangulado, conforman un conjunto característico de los sitios de la Península Tingitana, siendo documentados en el área de Tánger y cabo Achakar (Gilman, 1975), Gar Cahal (Vijande et al., 2008), Kef Boussaria, Oued Tahadart y KTG.

Como sucede en las cuevas de Magharat el-Khil y cabo Achakar, se pone de manifiesto la aparente coincidencia temporal de dicho clúster (Daugas et al., 2008), encabezado por el grupo Cardial y las acanaladas. Como ya adelantamos, la combinación de ambas técnicas en un mismo vaso constituye sin duda un argumento de peso, conformando ambas una estructura decorativa -grupos temáticos- muy similar, partiendo de impresiones verticales en el labio, seguidas de bandas horizontales arrastradas en el caso de la valva y acanaladuras en el caso de la cypraea, con frecuencia intercaladas con campos de impresiones verticales, aisladas o basculantes en el caso del grupo Cardial y aisladas o acompañadas por acanaladuras cortas en el caso de las acanaladas. Como puede verse en publicaciones precedentes ${ }^{8}$, las formas que comparten ambos grupos son esencialmente las mismas.

La concordancia radiocarbónica, bien establecida a partir de muestras domésticas de vida corta, animales y vegetales, situaría con probabilidad estos conjuntos en torno a 5475-5300 cal AC (Martínez et al., 2021). Así, dichas cerámicas cuentan con una mayor presencia en las UUEE 1103-3 y 1204-2, correspondientes a un Neolítico Antiguo bien respaldado por dataciones radiocarbónicas. El resto de los grupos decorativos propios de esta fase resultan más difíciles de ajustar, resultado de su menor presencia y su más compleja caracterización. Las incisas, vista su diversidad, podrían considerarse un grupo cronológicamente ubicuo, estando presentes desde el inicio de la secuencia asociada a motivos reticulados - quadrillé-. Muy distinto es el caso de la cerámica decorada a peine, la cual podría extenderse en conjunto dentro de un rango temporal más reciente. Así, el uso de peine basculante se encuentra presente desde el inicio de la secuencia neolítica en Ifri

$8 \quad$ El Idrissi, op. cit. n. 2.
Oudadane, compartiendo espacio con la cardial, desde el 5200 -Early Neolithic A/B-, si asumimos esa data como límite del inicio de dicha fase en este yacimiento (Morales et al., 2016). En los yacimientos del entorno de Tánger, un grupo de cerámicas con impresión y arrastre a peine -normalmente en la franja del borde- fueron incluidas en una fase avanzada dentro del Neolítico, en vista de su distribución estratigráfica (Gilman, 1975). Nosotros, en la cueva de Khil c, asumimos estas mismas producciones con probabilidad dentro de la primera mitad del v milenio -Early Neolithic B-, asociadas a una datación incluida en dicho rango -Beta-331847, $5790 \pm 30$ вр- (Martínez et al., 2018a), si bien en el caso de KTG quedarían pendientes de probar cronologías más elevadas, considerando el posible auge de las decoraciones a peine hacia el final del vi milenio como pondrían de manifiesto los conjuntos de Ifri Oudadane, sin descartar perduraciones dentro de la primera mitad del v milenio cal AC.

En este sentido, carecemos de dataciones absolutas o unidades estratigráficas que pudieran relacionarse de forma inequívoca con la primera mitad del $\mathrm{v}$ milenio, lo cual ayudaría a clarificar la génesis o primera aparición de las cerámicas de tipo Achakar. La secuencia obtenida no ayuda a resolver esta cuestión concreta. La presencia abundante de fragmentos impresos, principalmente cardiales y acanalados de origen indudablemente residual, en los niveles atribuidos a la fase media, oscurece nuestras conclusiones al respecto. Siguiendo las observaciones realizadas en otras localizaciones del área de Tánger y el Temara, como Magharat el Khil, El Kiffen o la necrópolis de Skhirat (Daugas, 2010; Turek y Vintr, 2016; Martínez et al., 2018a), su aparición podría no ser anterior a la mitad del v milenio cal AC. A este respecto, quedaría probada la coexistencia de tradiciones cerámicas impresas muy diferentes de la propia Ashakar Ware (Martínez et al., 2018a), al menos en el área de Melilla-Alhucemas -Al-Hoceima-, visibles en los sitios de Zafrin (Rojo et al., 2009) o Ifri Oudadane (Linstadter y Wagner, 2013). Estas tradiciones impresas tardías, que también comportan el uso de la concha estriada y lisa si bien, con motivos y disposiciones de aspecto diferenciado, 
podrían relacionarse con la aparente perduración de motivos impresos de aspecto cardial con cronologías avanzadas en otros yacimientos del Rif Oriental (Nekkal et al., 2015). En cualquier caso, en esta última región también se observan ciertas analogías con tradiciones saharianas que podrían situarse en la primera mitad del v milenio, como en el caso de Hassi Ouenzga (Linstädter, 2003) o algún ejemplo de wavy line en la fase ENB de Ifri Oudadane (Linstadter y Wagner, 2013).

\subsection{Origen de las primeras cerámicas del extremo noroccidental africano}

En cuanto al origen de las primeras cerámicas de la Península Tingitana, punto clave del debate al que aludimos al inicio de este trabajo, contamos con argumentos para defender la existencia de fenómenos de transmisión a través de contacto marítimo desde el Arco Latino europeo. Como han trazado diversos investigadores y modelizaciones (Isern et al., 2017), las primeras cerámicas, junto al resto del paquete neolítico constituido por animales $\mathrm{y}$ vegetales domesticados, arriban al $\mathrm{N}$ de Marruecos una vez alcanzada la Península Ibérica. Como en el caso de esta región (Pardo et al., 2019), las técnicas decorativas que presentan habrían sido en su mayor parte heredadas del mundo impresso-cardial del Mediterráneo noroccidental. Junto al uso de la concha dentada y su empleo preferente para realizar basculaciones -rocker-, el de otros soportes malacológicos como el canal sifonal de ciertos gasterópodos -fundamentalmente cypraea-, así como las digitaciones, ungulaciones y aplicaciones plásticas basadas en el uso de cordones, y sobre los que con frecuencia se observan impresiones cardiales seriadas, cuentan con precedentes en las primeras fases cardiales -Formativas o Fase I- del País Valenciano y Cataluña -c. 5600-5400 cal AC- (Bernabeu, 1989; Soler et al., 2011; Bernabeu et al., 2011; Oms, 2017), así como en producciones impresse anteriores del Mediterráneo central, costa tirrénica y adriática -5900-5500 cal AC- (Brandaglia, 1991; Radi et al., 2017; Podrug et al., 2018).
En este sentido, su aspecto arcaizante también vendría dado por cierta falta de estructuración y complejidad en las decoraciones y grupos temáticos, no detectándose el uso del natis para realizar motivos impresos. Las composiciones complejas parecen estar ausentes, así como las de tipo figurativo -véase antropomorfos-, primando en su lugar frisos colgantes conformados por acanaladuras (Ramos et al., 2008), mosaicos o campos abigarrados de impresiones, a veces cubriendo gran parte del cuerpo de la pieza y donde destaca el gusto por la decoración basculante, así como el uso de cordones de refuerzo, comunes en los yacimientos antiguos ibéricos e italianos. La ausencia de motivos impresos tipo boquique o sillon d'impressions, propios de la Impressa ligur -5850-5700 cal AC- (Manen et al., 2019), separa las producciones marroquíes de este círculo cultural concreto, que también se ha venido a documentar en el País Valenciano (Bernabeu et al., 2009). Ello plantea la idea de su transmisión a partir de grupos diferenciados de este último, identificados con el ámbito impresso-cardial, y probablemente ya establecidos en la costa andaluza del mar de Alborán entre 5600 y 5500 cal AC. Analogías significativas se observan en las decoraciones con presencia de arrastre cardial asociado a basculaciones o impresiones sencillas con la misma valva, intercaladas en bandas horizontales en distintos emplazamientos del ámbito catalán, como en la Cova del Toll (Cebriá et al., 2014), Cova Bonica o Guixeres (Daura et al., 2019; Oms, 2014). En este sentido, la ausencia de tratamientos a la almagra en estos primeros conjuntos marroquíes quizá deba explicarse por haberse producido dicho evento transcontinental en momentos anteriores a la generalización de esta técnica, que terminaría por convertirse en uno de los rasgos más reconocibles del denominado Neolítico Andaluz. Tampoco cabría excluir la posibilidad de una transmisión protagonizada por grupos ajenos a este ámbito cultural concreto, como podría ser el caso del territorio catalán o valenciano.

La aparición de formas cerámicas diferenciadas y muy particulares, como son vasos profundos de fondo cónico y cuello estrangulado con perfil exvasado, formas aparentemente divergentes de las habituales en 
las producciones cerámicas del vi milenio cal AC en el Mediterráneo europeo, se han relacionado en ocasiones con formas presentes en Andalucía dentro del horizonte propio del Neolítico Andaluz (Linstädter et al., 2012; Manen et al., 2007). Sin embargo, existen analogías similares en los vasos de borde exvasado y/o cordón impreso en la línea del cuello, presentes en algunos conjuntos típicamente cardiales del País Valenciano y Cataluña, como Benamer (García et al., 2015) y Sarsa en Alicante -vasos 321, 394- (García, 2017) o Guixeres de Vilobí en El Penedés -vaso 13- (Oms, 2014), siendo los fondos apuntados cada vez más reconocibles en estas primeras producciones cerámicas peninsulares. Formas similares, aunque raras, no son desconocidas en la impressa tirrénica (Brandaglia, 1991; Fugazzola et al., 2002). En cualquier caso, el desarrollo de nuevas formas cerámicas, modificaciones en las técnicas decorativas y la pérdida o simplificación de los motivos constituyen fenómenos esperables en un proceso de difusión transcontinental. En un evento caracterizado por la traslocación de un número limitado de personas a un nuevo territorio, la dificultad de mantener contactos fluidos con el grupo originario y la posible interacción con grupos locales, entre otros factores, acelerarían los procesos de mutación visibles en la cultura material del registro arqueológico.

\section{Conclusiones}

A la luz de datos actuales, la aparición en la Península Tingitana de estas primeras cerámicas tendría lugar en fechas próximas al c. 5500/5450 cal AC, proceso documentado a través de su asociación con cereales datados (Martínez et al., 2018b), lo que convertiría a Kaf Taht el-Ghar en el yacimiento con las cerámicas de mayor antigüedad hasta la fecha en el n de Marruecos y el conjunto del África mediterránea. Las necesarias exigencias de higiene radiocarbónica y un análisis crítico de las cronologías del Magreb no detectan de momento cerámicas en el Rif Oriental y Atlas Medio antes de 5200/5100 cal AC, frente a lo apoyado por otros autores (Dunne

Ediciones Universidad de Salamanca / @@®ఠ et al., 2020; Linstädter et al., 2016). De la misma manera, ha sido cuestionada su presencia en el Tell y Cap Bon con anterioridad al v milenio cal AC (Zilhão, 2014). En cualquier caso, la aparición de la tecnología cerámica en el Magreb occidental en cronologías anteriores, si bien podría ser considerada plausible y probable vista su difusión en el territorio del Sahara, aún no ha podido ser probada de forma fehaciente hasta la fecha.

El análisis de las cerámicas correspondientes al Neolítico Antiguo en este yacimiento ha permitido establecer paralelos dentro del complejo impresso-cardial con precedentes en el Mediterráneo centro-occidental europeo. Estos se encuentran fundamentalmente en el uso de conchas estriadas y lisas para realizar impresiones sencillas y basculantes, así como el arrastre cardial. Otras características son el empleo de gasterópodos, el uso de aplicaciones plásticas a base de cordones, impresiones digitadas y pellizcadas, así como el uso de matrices dentadas para realizar impresiones sencillas y basculantes. Todo ello cuenta con paralelos estrechos en los primeros conjuntos considerados cardiales en Cataluña - previos al denominado Cardial Estructurado o Franco-Ibérico- (Oms, 2014) y los conjuntos considerados arcaicos del Cardial Valenciano, no descartando el papel que una previsible facies de tipo arcaico en el sur de la Península pudo cumplir en la transmisión de esta tecnología a la costa magrebí. La ausencia de boquique y engobe a la almagra en estas primeras producciones de la Península Tingitana parece desvincularla tanto de la impressa del País Valenciano, heredera de la impressa ligur caracterizada por la técnica de sillon d'impressions $-c$. 5850-5700 cal AC-, como de las primeras almagras andaluzas (a partir de $c .5400 \mathrm{cal} \mathrm{AC}$ ), lo cual parece reforzar un evento de transmisión dentro de una ventana temporal situada entre ambas tradiciones.

La teórica influencia en las primeras producciones cerámicas del $\mathrm{N}$ de Marruecos, de estilos y técnicas cerámicas preexistentes en el territorio del Sahara entre grupos epipaleolíticos, si bien constituye una posibilidad digna de ser explorada, necesita de pruebas que puedan servir de argumento a su defensa. Así, la existencia de técnicas de tratamiento 
y decoración de evidente raigambre sahariana -uso de engobes colorantes, gusto por la decoración impresa a base de roulettes de cuerda enrollada- parece constituir una tradición más reciente en esta región. Si bien decoraciones de este tipo se han documentado en otras partes del Magreb mediterráneo, incluyendo el Rif oriental y Túnez, será el denominado grupo de la Ashakar-Skhirat Ware el que adquirirá un protagonismo especial en el Marruecos atlántico y Península Tingitana a partir de mediados del v milenio cal AC (Martínez et al., 2018a).

\section{Bibliografía}

Alday, A. y Moral, S. (2011): "El dominio de la cerámica Boquique: discusiones técnicas y cronoculturales". En Bernabeu, J.; Rojo, M. A. y Molina, L. (eds.): Las primeras producciones cerámicas: el VI milenio cal AC en la Península Ibérica. Valencia: Univ. de Valencia, pp. 65-80.

Bernabeu, J. (1989): La tradición cultural de las cerámicas impresas en la zona oriental de la Península Ibérica. Valencia: Diput. Prov. de Valencia.

Bernabeu, J.; Gómez, O.; Molina, L. y García, P. (2011): "La cerámica neolítica durante el vi milenio cal AC en el Mediterráneo central peninsular". En Bernabeu, J.; Rojo, M. A. y Molina, L. (eds.): Las primeras producciones cerámicas: el VI milenio cal $A C$ en la Peninsula Ibérica. Valencia: Univ. de Valencia, pp. 153-178.

Bernabeu, J.; Molina, L.; Esquembre, M. A.; OrteGA, J. R. y Boronat, J. R. (2009): "La cerámica impresa mediterránea en el origen del Neolítico de la Península Ibérica". En De Méditerranée et d'ailleurs... Mélanges offerts à J. Guilaine. Toulouse: Collège de France, pp. 83-95.

Brandaglia, M. (1991): "Il Neolitico a ceramica impressa dall'isola del Giglio. La ceramica”, Studi per l'ecologia del Quaternario, 13, pp. 43-104.

Camps-Fabrer, H. (1966): Matière et Art Mobilier dans la Préhistoire Nord-Africaine et Saharienne. Algers: Conseil de la Recherche Scientifique en Algérie.

Camps, G. (1969): Amekni. Néolithique Ancien du Hoggar. Paris: Arts et métiers graphiques.

Camps, G. (1974): Les civilisations préhistoriques de l'Afrique du Nord et du Sahara. Paris: CNRs.
Cebriá, A.; Oms, F. X.; Fontanalls, M.; Rodríguez, A.; Martín, P.; Soto, M.; Morales, J. I. y Vergès, J. M. (2014): "Nuevos datos para el Neolítico antiguo en el nordeste de la Península Ibérica procedentes de la Cova del Toll (Moià, Barcelona) y de la Cova de la Font Major (L'Espluga de Francolí, Tarragona)", Trabajos de Prehistoria, 71 (1), pp. 134-145.

Daugas, J.-P. (2010): "Le Néolithique du Maroc, 25 ans de coopération franco-marocaine", Les nouvelles de l'archéologie, 120-121, pp. 116-121.

Daugas, J.-P.; El Idrissi, A.; Ballouche, A.; Marinval, P. y Ouchaou, B. (2008): "Le Néolithique ancien au Maroc septentrional", Bulletin de la Société Préhistorique Française, 105, pp. 787-812.

Daura, J.; Sanz, M.; Oms, F. X.; Pedro, M.; Martínez, P.; Mendiela, S.; Oliva, M.; Gibaja, J. F.; Mozota, M.; Alonso-Eguiluz, M.; Albert, R. M.; Allue, E.; Bañuls-Cardona, S.; López-García, J. M.; Santos, F. J. y Fullola, J. M. (2019): “Deciphering Neolithic activities from a Cardial burial site (Cova Bonica) on the western Mediterranean Coast", Journal of Archaeological Science, 23, pp. 324-347.

Dunne, J.; Manning, K.; Linstädter, J.; Mikdad, A.; Breeze, P.; Hutterer, R.; Lehnig, S.; Morales, J.; Gillard, J.; Drake, T. y Evershed, R. P. (2020): "Pots, plants and animals: broad-spectrum subsistence strategies in the Early Neolithic of the Moroccan Rif region", Quaternary International, 555, pp. 96109.

Dunne, J.; Mercuri, A. M.; Evershed, R. P.; Bruni, S. y Di Lernia, S. (2016): "Earliest direct evidence of plant processing in prehistoric Saharan pottery", Nature Plants, 3 (1), 16194.

El Idrissi, A. (2001): Néolithique ancien du Maroc Septentrional dans son contexte régional. Rabat: Institut National de Sciences de l'Archéologie et du Patrimoine.

El Idrissi, A. (2008): "Caractérisation du Néolithique ancien de Kahf Boussaria”. En Bernal, D.; RaissouNI, B.; Ramos, J.; ZouaK, M. y Parodi, M. (eds.): En la orilla africana del Círculo del Estrecho. Historiografía y proyectos actuales. Cádiz: Univ. de Cádiz, pp. 397-423.

El Idrissi, A. (2012a): "La Céramique (El-Mnasra)”. En El Hajraoui, M. A.; Nespoulet, R.; Debénath, A. y Dibble, H. L. (eds.): Préhistoire de la Région de Rabat-Témara. Rabat: INSAP, pp. 150-153.

El Idrissi, A. (2012b): "La Céramique (El Harhoura 2)". En El Hajraoui, M. A.; Nespoulet, R.; 
60 R. M. Martínez Sánchez, J. C. Vera Rodríguez, G. Pérez Jordà y Leonor Peña-Chocarro / La decoración cerámica en el Neolítico...

Debénath, A. y Dibble, H. L. (eds.): Préhistoire de la Région de Rabat-Témara. Rabat: INSAP, pp. 77-81.

El IDRIssi, A. (2012c): "Le Néolithique du Maroc: état de la question", Rubricatum, 5, pp. 333-342.

Fugazzola, M. A.; Pessina, A. y Tiné, V. (2002): Le ceramiche impresse nel Neolitico Antico. Italia e Mediterraneo. Roma: Istituto Poligrafico e Zecca dello Stato.

García, G.; Torregrosa, P.; Jover, F. J. y López, E. (2015): "La ocupación del Neolítico antiguo cardial de Benàmer (Muro de l'Alcoi, Alicante)". En Gonçalves, V. S.; Diniz, M. y Sousa, A. C. (eds.): Actas do 5. ${ }^{\circ}$ Congresso do Neolitico Peninsular. Lisboa: UNIARQ, PP. 131-138.

García, P. (2017): Las cerámicas neolíticas de la Cova de la Sarsa (Bocairent, Valencia). Tipología, estilo e identidad. Valencia: MUPREVA.

Gilman, A. (1975): A Later Prehistory of Tangier, Morocco. Cambridge: Peabody Museum-Harvard University.

Isern, N.; Zilhão, J.; Fort, J. y Ammerman, A. J. (2017): "Modeling the role of voyaging in the coastal spread of the Early Neolithic in the West Mediterranean", PNAS, 114, pp. 897-902.

Jodin, A. (1958-1959): "Les grottes d'El Kril à Achakar, province de Tanger", Bulletin d'Archéologie Marocaine, III, pp. 249-313.

JóRdeczKa, M.; Królik, H.; Masojc, M. y Schild, R. (2011): "Early Holocene pottery in the Western Desert of Egypt: new data from Nabta Playa", Antiquity, 85, pp. 99-115.

Linstädter, J.; Medved, I.; Solich, M. y Weniger, G.-C. (2012): "Neolithisation process within the Alboran territory: Models and possible African impact”, Quaternary International, 274, pp. 219-232.

Linstädter, J. y Wagner, G. (2013): “The early Neolithic pottery of Ifri Oudadane, Ne Morocco-Qualitative and quantitative evidence", Journal of African Archaeology, 11, pp. 155-196.

Manen, C. (2000): Le Néolithique ancien entre Rhône et Èbre: analyse des céramiques décorées. Toulouse: École des Hautes Études en Sciences Sociales.

Manen, C.; Marchand, G. y Carvalho, A. F. (2007): "Le Néolithique ancien de la Péninsule Ibérique : vers une nouvelle évaluation du mirage africain?". En Évin, J. (ed.): Actes XXVI Congrès Préhistorique de France. Congrès du Centenaire. Un siècle de construction du discours scientifique en Préhistoire. Avignon: SPF, pp. 133-151.

Ediciones Universidad de Salamanca / @@
Manen, C.; Perrin, T.; Raux, A.; Binder, D.; Le Bourdonnec, F.-X.; Briois, F.; Convertini, F.; Dubernet, S.; Escallon, G.; Gomart, L.; Guilaine, J.; Hamon, C.; Philibert, S. y Queffelec, A. (2019): "Le sommet de l'iceberg ? Colonisation pionnière et néolithisation de la France méditerranéenne", Bulletin de la Société Préhistorique Française, 116, pp. 317-361.

Martínez, R. M.; Vera, J. C.; Moreno, M.; Pérez, G.; Peña-Chocarro, L. y Воквот, Y. (2017): "Beyond Adornment: Cowry Use as Potter's Tool in the First Impressed Wares of the Southwestern Mediterranean Coast (Northern Morocco)", Journal of Island and Coastal Archaeology, 13 (3), pp. 420-437.

Martínez, R. M.; Vera, J. C.; Peña-Chocarro, L.; Воквот, Y.; Pérez, G. y Pardo, S. (2018a): "The Middle Neolithic of Morocco's North-Western Atlantic Strip: New Evidence from the El-Khil Caves (Tangier)", African Archaeological Review, 35, pp. 417-442.

Martínez, R. M.; Vera, J. C.; Pérez, G.; Moreno, M.; Воквот, Y. у PeÑa-Chocarro, L. (2021): "Revisiting the Epipalaeolithic-Neolithic Transition in the Extreme Nw of Africa: The Latest Results of the Chronological Sequence of the Cave of Kaf Taht el-Ghar (Tétouan, Morocco)", African Archaeologica Review. https://doi.org/10.1007/s10437-02109425-x

Martínez, R. M.; Vera, J. C.; Pérez, G.; Peña-ChoCARro, L. y Воквот, Y. (2018b): "The beginning of the Neolithic in north-western Morocco", Quaternary International, 470 (в), pp. 485-496.

Morales, J.; Pérez, G.; Peña-Chocarro, L.; Вokhbot, Y.; Vera, J. C.; Martínez, R. M y LinstäDter, J. (2016): "The introduction of South-Western Asian domesticated plants in North-Western Africa: an archaeobotanical contribution from Neolithic Morocco", Quaternary International, 412, pp. 96-109.

Mulazzani, S.; Bellouchet, L.; Salanova, L.; Auadi, N.; Dridi, Y.; Eddargach, W.; Morales, J.; Tombret, O.; Zazzo, A. y Zoughlami, J. (2016): "The emergence of the Neolithic in North Africa: A new model for the Eastern Maghreb", Quaternary International, 410, pp. 123-143.

Nekkal, F.: LinstäDter, J.; Aouraghe, H. y BougariaNE, B. (2015): "Ifri Ouzabour: L'extrême extension de la culture cardiale sur le littoral méditerranéen sud (Rif oriental, Maroc)", Bulletin de l'Institut Scientif-que, Rabat, Section Sciences de la Terre, 37, pp. 55-76. 
Oмs, F. X. (2014): La neolitització del Nord-Est de la P. Ibèrica à partir de les datacions de Carboni 14 i les primeres ceràmiques impreses c. 5600-4600 cal BC. Barcelona: Univ. de Barcelona.

Oms, F. X.; Terradas, X.; Morell, B. y Gibaja, J. F. (2017): "Mesolithic-Neolithic transition in the northeast of Iberia: Chronology and socioeconomic dynamics", Quaternary International, 470 (в), pp. 383-387.

Orton, C. y Hughes, M. (2013): Pottery in Archaeology. Cambridge: cup.

Pardo, S.; García, D. y Bernabeu, J. (2019): "Evidences of branching and blending phenomena in the pottery decoration during the dispersal of the Early Neolithic across Western Europe", Journal of Archaeological Science, 23, pp. 252-264.

Podrug, E.; McClure, S. B.; Perhoc, Z.; Kacar, S.; Reed, K. y Zavodny, E. (2018): "Rašinovac near Ždrapanj (Northern Dalmatia)-An Early Neolithic site", Archaeologia Adriatica, 12, pp. 47-97.

Radi, G. y Petrinelli, C. (2017): "The beginning of the Neolithic era in Central Italy", Quaternary International, 470 (в), pp. 270-284.

Ramos, J.; Zounk, M.; Bernal, D. y Raissouni, B. (2008): Las ocupaciones humanas de la cueva de Caf Taht el-Ghar (Tetuán). Los productos arqueológicos en el contexto del Estrecho de Gibraltar. Cádiz: Univ. de Cádiz.

Rojo, M.; Garrido, R.; Belllver, J. A.; Bravo, A.; García, I.; Gámez. S. y Tejedor, C. (2009): Zafrín. Un asentamiento del Neolitico Antiguo en las islas Chafarinas (Norte de África, España). Valladolid: Univ. de Valladolid.

Roubet, C. (1979): Économie pastorale préagricole en Algérie Orientale. Le Néolithique de tradition capsienne. Exemple: L'Aurès. Paris: CNRS.

Soler, J. A.; Gómez, O.; García, G. y Roca de TogoRES, C. (2011): "Sobre el primer horizonte neolítico en la Cova d'En Pardo (Planes, Alicante). Su evaluación desde el registro cerámico". En Bernabeu, J.; Rojo, M. A. y Molina, L. (eds.): Las primeras producciones cerámicas: el VI milenio cal AC en la Península Ibérica. Valencia: Univ. de Valencia, pp. 201-212.

Stempfle, S.; Linstädter, J.; Nickel, K. G.; Mikdad, A. y Schмidt, P. (2018): "Early Neolithic pottery of Ifri n'Etsedda, NE-Morocco-Raw materials and fabrication techniques", Journal of Archaeological Science, 19, pp. 200-212.

TARradell, M. (1954): "Noticia sobre la excavación de Gar Cahal", Tamuda, II, pp. 344-358.

TARradell, M. (1955): "Avance de la primera campańa de excavaciones en Caf Taht el Gar", Tamuda, III, pp. 307-322.

Tarradell, M. (1957-1958): "Caf Taht el Gar, cueva neolítica en la región de Tetuán (Marruecos)”, Ampurias, 19-20, pp. 137-166.

TureK, J. y Vintr, J. (2016): "Neolit Maghrebu ve světle nových radiokarbonových dat", Zivá Archeologie, 18 , pp. 10-15.

Vijande, E.; Ramos, J.; Zouak, M.; Cantillo, J.; El Idrissi, A.; Domínguez-Bella, S. y MaAte, A. (2011): "Revisión de los productos arqueológicos de la cueva de Gar Cahal depositados en el Museo Arqueológico de Tetuán”. En Bernal, D.; Raissouni, B.; Arcila, M.; Youbi, M.; Ramos, J.; ZouaK, M.; López, J. A.; Maatouk, M.; El Kheyari, A.; El Moumni, B.; Ghottes, M. y Azzariohi, A. (eds.): Arqueología y turismo en el Círculo del Estrecho. Estrategias para la puesta en valor de los recursos patrimoniales del Norte de Marruecos. Cádiz: Univ. de Cádiz, pp. 265-287.

ZILHÃo, J. (2014): "Early prehistoric navigation in the western Mediterranean: implications for the Neolithic transition in Iberia and the Maghreb", Eurasian Prehistory, 11, pp. 185-200. 